
by/4.0/), which permits unrestricted re-use, distribution, and reproduction in any medium, provided the original work is properly cited.

\title{
Sharp endpoint estimates for some operators associated with the Laplacian with drift in Euclidean space
}

\author{
Hong-Quan Li and Peter Sjögren
}

Abstract. Let $v \neq 0$ be a vector in $\mathbb{R}^{n}$. Consider the Laplacian on $\mathbb{R}^{n}$ with drift $\Delta_{v}=\Delta+2 v \cdot \nabla$ and the measure $d \mu(x)=e^{2\langle v, x\rangle} d x$, with respect to which $\Delta_{v}$ is self-adjoint. This measure has exponential growth with respect to the Euclidean distance. We study weak type $(1,1)$ and other sharp endpoint estimates for the Riesz transforms of any order, and also for the vertical and horizontal Littlewood-Paley-Stein functions associated with the heat and the Poisson semigroups.

\section{Introduction}

Consider the weighted manifold $\mathbb{R}^{(n, v)}$, defined as $\mathbb{R}^{n}$ with the Euclidean distance and the measure $d \mu(x)=e^{2\langle v, x\rangle} d x$. Here, $v=\left(v_{1}, \ldots, v_{n}\right) \in \mathbb{R}^{n} \backslash\{0\}$ is fixed and $\langle\cdot, \cdot\rangle$ denotes the inner product on $\mathbb{R}^{n}$. Notice that large balls in this space have exponential volume growth. With $\mathbb{R}^{(n, v)}$, we associate the Laplacian with drift

$$
\Delta_{v}=\sum_{i=1}^{n}\left(\frac{\partial^{2}}{\partial x_{i}^{2}}+2 v_{i} \frac{\partial}{\partial x_{i}}\right) .
$$

We denote by $\nabla$ the usual gradient operator in $\mathbb{R}^{n}$. Notice that the Green formulas hold with respect to the measure $\mu$; that is,

$$
\int_{\mathbb{R}^{n}} f \Delta_{v} w d \mu=-\int_{\mathbb{R}^{n}}\langle\nabla f, \nabla w\rangle d \mu=\int_{\mathbb{R}^{n}} w \Delta_{v} f d \mu,
$$

provided $f$ and $w$ are smooth and $f$ or $w$ has compact support. Thus, $\Delta_{v}$ has a selfadjoint extension in $L^{2}\left(\mathbb{R}^{n}, d \mu\right)$, also denoted $\Delta_{v}$.

We will consider Riesz transforms and Littlewood-Paley-Stein functions of any order in $\mathbb{R}^{(n, v)}$. These operators are defined and studied in many general settings, such as Lie groups, symmetric spaces, and other Riemannian manifolds. Their $L^{p}$ boundedness properties for $1<p<\infty$ have been well studied and are known in several cases. In particular, the classical results are not always valid on manifolds. We refer the

Received by the editors October 24, 2019; revised March 27, 2020.

Published online on Cambridge Core June 16, 2020.

H.-Q. Li is partially supported by NSF of China (Grants No. 11625102 and No. 11571077) and The Program of Shanghai Academic Research Leader (18XD1400700). Both authors profited from a grant from the Gothenburg Centre for Advanced Studies in Science and Technology.

AMS subject classification: 42B20, 42B25, 58J35.

Keywords: Riesz transform, Littlewood-Paley-Stein operators, Heat semigroup, Laplacian with drift. 
reader to $[1,5-12,18,21-26]$ and references therein. Interesting counterexamples can be found in $[7-9,18]$ for Riesz transforms of order one, and in $[1,12,14]$ for those of order two. The setting of the Ornstein-Uhlenbeck semigroup in Euclidean space is considered in [13, 27]; see also [2]. There the measure is Gaussian, thus finite, but not doubling.

The weak type $(1,1)$ property of these operators is more difficult and less known in spaces of exponential volume growth. The main reason is that the existing methods to treat singular integrals are insufficient. Theorem 1.2 of [9] is a weaker estimate. But some results have been established; see $[14,28]$ for the affine group, which is a typical case without spectral gap. Some other groups and spaces with no gap are treated in [17]. In [3], Anker proved the weak type $(1,1)$ of first- and second-order Riesz transforms in a symmetric space of the non-compact type. This case is subtler because of the spectral gap. See also [4] for other examples. Further, $[19,20]$ deal with the Laplacian with drift.

Returning to our setting $\mathbb{R}^{(n, v)}$, we mention that Lohoué and Mustapha [26] proved that the Riesz transforms $\nabla^{k}\left(-\Delta_{v}\right)^{-k / 2}$ of any order $k$ are bounded on $L^{p}, 1<p<$ $\infty$. Their setting and results are actually more general. In [20], the authors and Y.-R. Wu showed that the first-order Riesz transform $\nabla\left(-\Delta_{v}\right)^{-1 / 2}$ is of weak type $(1,1)$ in $\mathbb{R}^{(n, v)}$. Here and in the sequel, $L^{p}$ and weak $L^{p}$ estimates in $\mathbb{R}^{(n, v)}$ always refer to the measure $\mu$.

For $\mathbb{R}^{(n, v)}$, we observe that $\partial / \partial x_{i}$ commutes with $\Delta_{v}$ and thus with any negative power of $-\Delta_{v}$, so that the factors $\nabla^{k}$ and $\left(-\Delta_{v}\right)^{-k / 2}$ can be written in any order. We will study the weak type $(1,1)$ property of $\nabla^{k}\left(-\Delta_{v}\right)^{-k / 2}$. But instead of $\nabla^{k}$, we will use a general homogeneous differential operator of order $k \geq 1$,

$$
D=\sum_{|\alpha|=k} a_{\alpha} \partial^{\alpha}
$$

with constant coefficients, not all 0 . Our Riesz operator will thus be

$$
R_{D}=D\left(-\Delta_{v}\right)^{-\frac{k}{2}}
$$

Letting $\partial_{v}$ denote differentiation along the vector $v$, we can write $D$ as a sum

$$
D=\sum_{i=0}^{k} \partial_{v}^{i} D_{k-i}^{\prime},
$$

where $D_{k-i}^{\prime}$ is a constant coefficient operator of order $k-i$ involving only differentiation in directions orthogonal to $v$. The maximal order of differentiation along $v$ is then

$$
q=\max \left\{i: D_{k-i}^{\prime} \neq 0\right\} \in\{0, \ldots, k\},
$$

and this quantity turns out to be significant.

Our result about $R_{D}$ is the following theorem.

Theorem 1.1 With $D$ and $q$ as just described, the Riesz transform $R_{D}=D\left(-\Delta_{v}\right)^{-k / 2}$ is of weak type $(1,1)$ if and only if $q \leq 2$. When $q \geq 3$, there exists a constant $C=C(v, D)$ 
such that for all $f \in L\left(1+\ln ^{+} L\right)^{\frac{q}{2}-1}(\mu)$ and all $\lambda>0$, we have

$$
\mu\left\{x ;\left|R_{D} f(x)\right|>\lambda\right\} \leq C \int \frac{|f|}{\lambda}\left(1+\ln ^{+} \frac{|f|}{\lambda}\right)^{\frac{q}{2}-1} d \mu .
$$

This inequality is sharp in the sense that q cannot be replaced by any smaller number.

Let $\left(e^{t \Delta_{v}}\right)_{t>0}$ denote the heat semigroup on $\mathbb{R}^{(n, v)}$, which is a symmetric diffusion semigroup in the sense of [29] (the conservation property can be justified by [16, Theorem 11.8], and the other properties are obvious). Further, let $\left(e^{-t \sqrt{-\Delta_{v}}}\right)_{t>0}$ denote the Poisson semigroup. For $f \in C_{0}^{\infty}$, we define the vertical Littlewood-Paley-Stein functions associated with the operator $D$ from (1.1) as

$$
\begin{aligned}
& \mathcal{H}_{D}(f)(x)=\left(\int_{0}^{+\infty}\left|t^{\frac{k}{2}} D e^{t \Delta_{v}} f(x)\right|^{2} \frac{d t}{t}\right)^{\frac{1}{2}}, \\
& \mathcal{G}_{D}(f)(x)=\left(\int_{0}^{+\infty}\left|t^{k} D e^{-t \sqrt{-\Delta_{v}}} f(x)\right|^{2} \frac{d t}{t}\right)^{\frac{1}{2}} .
\end{aligned}
$$

The $L^{p}(1<p<+\infty)$ boundedness of $\mathcal{G}_{D}$ and $\mathcal{H}_{D}$ is easy to verify.

\section{Theorem 1.2}

(i) The operator $\mathcal{H}_{D}$ is of weak type $(1,1)$ if and only if $q \leq 1$. When $q>1$, there exists a constant $C=C(v, D)$ such that for all $f \in L\left(1+\ln ^{+} L\right)^{\frac{q}{2}-\frac{3}{4}}(\mu)$ and all $\lambda>0$,

$$
\mu\left\{x ; \mathcal{H}_{D} f(x)>\lambda\right\} \leq C \int \frac{|f|}{\lambda}\left(1+\ln ^{+} \frac{|f|}{\lambda}\right)^{\frac{9}{2}-\frac{3}{4}} d \mu .
$$

(ii) The operator $\mathcal{G}_{D}$ is of weak type $(1,1)$ if and only if $q \leq 2$. When $q>2$, there exists a constant $C=C(v, D)$ such that for all $f \in L\left(1+\ln ^{+} L\right)^{\frac{q}{2}-1}(\mu)$ and all $\lambda>0$,

$$
\mu\left\{x ; \mathcal{G}_{D} f(x)>\lambda\right\} \leq C \int \frac{|f|}{\lambda}\left(1+\ln ^{+} \frac{|f|}{\lambda}\right)^{\frac{q}{2}-1} d \mu .
$$

In (1.3) and (1.4), q cannot be replaced by any smaller number.

Now consider the horizontal Littlewood-Paley-Stein functions and related maximal operators, defined for $f \in C_{0}^{\infty}$ by

$$
\begin{aligned}
& h_{k}(f)(x)=\left(\int_{0}^{+\infty}\left|t^{k} \frac{\partial^{k}}{\partial t^{k}} e^{t \Delta_{v}} f(x)\right|^{2} \frac{d t}{t}\right)^{\frac{1}{2}}, \\
& H_{k}(f)(x)=\sup _{t>0}\left|t^{k} \frac{\partial^{k}}{\partial t^{k}} e^{t \Delta_{v}} f(x)\right|, \\
& g_{k}(f)(x)=\left(\int_{0}^{+\infty}\left|t^{k} \frac{\partial^{k}}{\partial t^{k}} e^{-t \sqrt{-\Delta_{v}}} f(x)\right|^{2} \frac{d t}{t}\right)^{\frac{1}{2}}, \\
& G_{k}(f)(x)=\sup _{t>0}\left|t^{k} \frac{\partial^{k}}{\partial t^{k}} e^{-t \sqrt{-\Delta_{v}}} f(x)\right| .
\end{aligned}
$$

Here, $k \geq 1$ for $h_{k}$ and $g_{k}$, but $k \geq 0$ for $H_{k}$ and $G_{k}$.

These operators are bounded on $L^{p}$ for $1<p<+\infty$ in the setting of a general symmetric semigroup with the contraction property; see [29]. The weak type $(1,1)$ property of $G_{k}, k \geq 0$, in a general setting was obtained in [19]. For the other three 
operators, we have the following endpoint estimates. We remark that the weak type $(1,1)$ of $H_{0}$ was obtained in [20, Theorem 2].

Theorem 1.3 The operators $h_{1}, H_{1}$, and $g_{k}$ with $k \geq 1$ are of weak type $(1,1)$. For $k \geq 2$, $h_{k}$ and $H_{k}$ are not of weak type $(1,1)$; however, there exists a constant $C=C(v, k)$ such that for all $f \in L\left(1+\ln ^{+} L\right)^{\frac{k}{2}-\frac{3}{4}}(\mu)$,

$$
\mu\left\{x ; h_{k} f(x)>\lambda\right\} \leq C \int \frac{|f|}{\lambda}\left(1+\ln ^{+} \frac{|f|}{\lambda}\right)^{\frac{k}{2}-\frac{3}{4}} d \mu \quad \forall \lambda>0,
$$

and for all $f \in L\left(1+\ln ^{+} L\right)^{\frac{k}{2}-\frac{1}{2}}(\mu)$,

$$
\mu\left\{x ; H_{k} f(x)>\lambda\right\} \leq C \int \frac{|f|}{\lambda}\left(1+\ln ^{+} \frac{|f|}{\lambda}\right)^{\frac{k}{2}-\frac{1}{2}} d \mu \quad \forall \lambda>0 .
$$

In these two estimates, the exponents cannot be replaced by any smaller numbers.

Our estimates, in particular those involving Orlicz spaces, are optimal and go beyond earlier known results.

The structure of this paper is as follows. Section 2 contains estimates for the kernels of the Riesz transforms, which are used in Section 3 to prove Theorem 1.1. A fundamental tool here is Proposition 3.1 that is also needed in the later sections. The two parts of Theorem 1.2 are proved in Sections 4 and 5. Section 6 gives estimates for the time derivatives of the heat kernel, which are applied in the proof of Theorem 1.3 in Section 7.

In this paper, $C$ denotes various constants that depend only on $n$ and $D$ in Sections 2-5, and only on $n$ and $k$ in Sections 6 and 7. By $A \lesssim B$, we mean $A \leq C B$ with such a $C$ (we say that $A$ is controlled by $B$ ), and $A \sim B$ stands for $A \leq C B$ and $B \leq C A$.

\subsection{Notation and Simple Facts}

We assume that $v=e_{1}=(1,0, \ldots, 0)$, which is no restriction; see [20]. Then $d \mu=$ $e^{2 x_{1}} d x$, and it will be convenient to write points in $\mathbb{R}^{n}$ as $x=\left(x_{1}, x^{\prime}\right) \in \mathbb{R} \times \mathbb{R}^{n-1}$. Further, we denote $\mathbb{N}=\{0,1,2, \ldots\}$ and $\mathbb{N}^{+}=\{1,2, \ldots\}$.

We have for any $x \in \mathbb{R}^{n}$ and $r \leq 1$,

$$
\mu(B(x, r)) \sim r^{n} e^{2 x_{1}} .
$$

It follows that our space has the local doubling property,

The heat kernel $p_{t}(x, y)$ in our setting is defined through

$$
e^{t \Delta_{e_{1}}} f(x)=\int p_{t}(x, y) f(y) d \mu(y), \quad t>0, \quad x \in \mathbb{R}^{n},
$$

for suitable functions $f$. It is explicitly given by ( $c f$. [16, p. 258])

$$
\begin{aligned}
p_{t}(x, y) & =(4 \pi t)^{-\frac{n}{2}} e^{-x_{1}-y_{1}} e^{-t} e^{-\frac{|x-y|^{2}}{4 t}} \\
& =(4 \pi t)^{-\frac{n}{2}} e^{-x_{1}-y_{1}-|x-y|} e^{-t\left(\frac{|x-y|}{2 t}-1\right)^{2}},
\end{aligned}
$$

for all $t>0$ and $x=\left(x_{1}, x^{\prime}\right), y=\left(y_{1}, y^{\prime}\right) \in \mathbb{R} \times \mathbb{R}^{n-1}$. 
Our results include the simpler case $n=1$, but the proofs are written for $n \geq 2$. Except for Proposition 3.1, we leave it to the reader to see how the arguments simplify for $n=1$.

\section{Estimates of the Riesz Kernels}

With $D, k$, and $q$ as in Theorem 1.1, we write $R_{D}(x, y)$ for the kernel of the Riesz transform $R_{D}=D\left(-\Delta_{e_{1}}\right)^{-k / 2}$. The multi-index $\alpha$ will be split as $\alpha=\left(\alpha_{1}, \alpha^{\prime}\right)$.

We state and prove local and global estimates for $R_{D}(x, y)$.

Proposition 2.1 The kernel $R_{D}(x, y)$ satisfies the local standard estimates

$$
\begin{aligned}
\left|R_{D}(x, y)\right| & \lesssim \frac{1}{\mu(B(x,|x-y|))}, & & 0<|x-y| \leq 1, \\
\left|\nabla_{y} R_{D}(x, y)\right| & \lesssim \frac{1}{|x-y| \mu(B(x,|x-y|))}, & & 0<|x-y| \leq 1 .
\end{aligned}
$$

Proposition 2.2 The kernel $R_{D}(x, y)$ satisfies

$$
\left|R_{D}(x, y)\right| \lesssim e^{-x_{1}-y_{1}-|x-y|}|x-y|^{\frac{q-n-1}{2}}\left[1+\left(\frac{\left|x^{\prime}-y^{\prime}\right|^{2}}{|x-y|}\right)^{\frac{k}{2}}\right], \quad|x-y|>1 .
$$

This estimate is sharp in the sense that there exists a ball $B \subset \mathbb{R}^{n-1}$, depending only on $n$ and $D$, such that

$$
(-1)^{k} R_{D}(x, y) \sim e^{-x_{1}-y_{1}-|x-y|}\left(x_{1}-y_{1}\right)^{\frac{q-n-1}{2}} \text { for } \frac{x^{\prime}-y^{\prime}}{\left(x_{1}-y_{1}\right)^{1 / 2}} \in B,
$$

if $x_{1}-y_{1}>0$ is large enough.

We will need the following simple integral estimate.

Lemma 2.3 With $v \in \mathbb{R}$ and $a>0$, let

$$
B_{v}(a)=\int_{0}^{+\infty} t^{v} e^{-t} e^{-\frac{a^{2}}{4 t}} \frac{d t}{t} .
$$

(i) Then for $a \leq 2$,

$$
B_{v}(a) \sim \begin{cases}a^{2 v} & \text { if } v<0 \\ \log \left(1+a^{-1}\right) & \text { if } v=0 \\ 1 & \text { if } v>0\end{cases}
$$

(ii) Moreover,

$$
B_{v}(a)=\sqrt{2 \pi} 2^{-v} a^{v-1 / 2} e^{-a}\left(1+O\left(a^{-1}\right)\right), \quad a \rightarrow \infty,
$$

and this estimate remains true if $B_{v}(a)$ is replaced by

$$
\int_{(a / 2-\ell) \vee 0}^{a / 2+\ell} t^{v} e^{-t} e^{-\frac{a^{2}}{4 t}} \frac{d t}{t}
$$

for any $\ell \geq a^{3 / 4}$.

In this lemma, the implicit constants depend on $v$ but not on a. 
Lemma 2.3 can be proved either with elementary estimates or by evaluating the integral in terms of Bessel functions according to the 12-th equality of [15, \$3.471]. The latter option also requires an easy estimate of those parts of the integral left out in the last statement.

We will also use a trivial extension of this lemma. Let $Q(t)=\sum_{v} q_{v} t^{v}$ be any finite linear combination of real powers of the variable $t>0$. Then Lemma 2.3(ii) implies that

$$
\int_{0}^{+\infty} Q(t) e^{-t} e^{-\frac{a^{2}}{4 t}} \frac{d t}{t}=\sqrt{2 \pi} Q(a / 2) a^{-1 / 2} e^{-a}\left(1+O\left(a^{-1}\right)\right), \quad a \rightarrow \infty .
$$

To prepare for the proofs of Propositions 2.1 and 2.2, we deduce an expression for $R_{D}(x, y)$. From (1.8), it follows that the kernel of $\left(-\Delta_{e_{1}}\right)^{-k / 2}$ is

$$
\begin{aligned}
\left(-\Delta_{e_{1}}\right)^{-\frac{k}{2}}(x, y) & =\frac{1}{\Gamma(k / 2)} \int_{0}^{+\infty} t^{\frac{k}{2}} p_{t}(x, y) \frac{d t}{t} \\
& =(4 \pi)^{-\frac{n}{2}} \frac{1}{\Gamma(k / 2)} e^{-x_{1}-y_{1}} \int_{0}^{+\infty} t^{\frac{k-n}{2}} e^{-t} e^{-\frac{|x-y|^{2}}{4 t}} \frac{d t}{t}
\end{aligned}
$$

Thus,

$$
R_{D}(x, y)=D_{x}\left(-\Delta_{e_{1}}\right)^{-\frac{k}{2}}(x, y)=\frac{1}{\Gamma(k / 2)} \int_{0}^{+\infty} t^{\frac{k}{2}} D_{x} p_{t}(x, y) \frac{d t}{t} .
$$

Recall that the Hermite polynomial of degree $j \in \mathbb{N}$ is defined by

$$
H_{j}(s)=(-1)^{j} e^{s^{2}} \frac{d^{j}}{d s^{j}} e^{-s^{2}},
$$

and $H_{\alpha}=\otimes_{i} H_{\alpha_{i}}$ for any multi-index $\alpha$. The $H_{\alpha}$ are orthogonal with respect to the Gaussian measure $e^{-|x|^{2}} d x$ in $\mathbb{R}^{n}$, and the leading term of $H_{j}(s)$ is $2^{j} s^{j}$.

The definition of $H_{\alpha}$ implies that

$$
\partial_{x}^{\alpha} e^{-\frac{|x-y|^{2}}{4 t}}=\left(-\frac{1}{2 \sqrt{t}}\right)^{|\alpha|} H_{\alpha}\left(\frac{x-y}{2 \sqrt{t}}\right) e^{-\frac{|x-y|^{2}}{4 t}} .
$$

The kernel $R_{D}(x, y)$ is a linear combination of terms $\partial_{x}^{\alpha}\left(-\Delta_{e_{1}}\right)^{-k / 2}(x, y)$, with $\alpha=\left(\alpha_{1}, \alpha^{\prime}\right)$ a multi-index of length $|\alpha|=k$ and with $\alpha_{1} \leq q$. When we take the $\partial_{x}^{\alpha}$ derivative of the expression in (1.8), some differentiations with respect to $x_{1}$ will fall on the factor $e^{-x_{1}}$, and some on the factor $e^{-|x-y|^{2} / 4 t}$. Those differentiations falling on $e^{-|x-y|^{2} / 4 t}$ will be given by a multi-index $\widetilde{\alpha}=\left(\widetilde{\alpha}_{1}, \alpha^{\prime}\right)$, with $0 \leq \widetilde{\alpha}_{1} \leq \alpha_{1}$. We then see from (2.6) and (2.8), with $\alpha$ replaced by $\widetilde{\alpha}$, that $\partial_{x}^{\alpha}\left(-\Delta_{e_{1}}\right)^{-k / 2}(x, y)$ will be a sum of terms proportional to

$$
\begin{aligned}
(-1)^{\alpha_{1}-\widetilde{\alpha}_{1}} e^{-x_{1}-y_{1}} \int_{0}^{+\infty} t^{\frac{k-n}{2}} e^{-t}\left(-\frac{1}{2 \sqrt{t}}\right)^{|\widetilde{\alpha}|} H_{\widetilde{\alpha}}\left(\frac{x-y}{2 \sqrt{t}}\right) e^{-\frac{|x-y|^{2}}{4 t}} \frac{d t}{t} \\
=(-1)^{|\alpha|} 2^{-|\widetilde{\alpha}|} e^{-x_{1}-y_{1}} \\
\quad \times \int_{0}^{+\infty} t^{\frac{\alpha_{1}-\widetilde{\alpha}_{1}-n}{2}} H_{\widetilde{\alpha}_{1}}\left(\frac{x_{1}-y_{1}}{2 \sqrt{t}}\right) H_{\alpha^{\prime}}\left(\frac{x^{\prime}-y^{\prime}}{2 \sqrt{t}}\right) e^{-t} e^{-\frac{|x-y|^{2}}{4 t}} \frac{d t}{t},
\end{aligned}
$$


where $\widetilde{\alpha}_{1}$ runs from 0 to $\alpha_{1}$. These terms have positive coefficients coming from Leibniz' formula and from the factors $(4 \pi)^{-n / 2}$ and $1 / \Gamma(k / 2)$.

Expanding the Hermite polynomials here, we obtain a sum of terms proportional to

$$
\pm e^{-x_{1}-y_{1}}\left(x_{1}-y_{1}\right)^{\gamma_{1}}\left(x^{\prime}-y^{\prime}\right)^{\gamma^{\prime}} \int_{0}^{+\infty} t^{\frac{\alpha_{1}-\widetilde{\alpha}_{1}-\gamma_{1}-\left|y^{\prime}\right|-n}{2}} e^{-t} e^{-\frac{|x-y|^{2}}{4 t}} \frac{d t}{t},
$$

and the sum is now taken also over a multi-index $\left(\gamma_{1}, \gamma^{\prime}\right)$ with $0 \leq \gamma_{1} \leq \widetilde{\alpha}_{1}$ and $0 \leq \gamma^{\prime} \leq$ $\alpha^{\prime}$ (componentwise ordering).

Proof of Proposition 2.1 Here, $|x-y| \leq 1$. If the exponent of $t$ in the integral in (2.10) is negative, we see from Lemma 2.3(i) that the modulus of the expression (2.10) is controlled by

$$
e^{-2 x_{1}}|x-y|^{\gamma_{1}+\left|\gamma^{\prime}\right|}|x-y|^{\alpha_{1}-\widetilde{\alpha}_{1}-\gamma_{1}-\left|\gamma^{\prime}\right|-n} \leq e^{-2 x_{1}}|x-y|^{-n} .
$$

For other values of the exponent, the bound $e^{-2 x_{1}}|x-y|^{-n}$ also follows. This is the first standard estimate (2.1). To obtain (2.2), it is enough to trace the argument given with a differentiation also in $y$.

Proof of Proposition 2.2 For $|x-y|>1$, Lemma 2.3(ii) implies that expression (2.10) equals constant times

$$
e^{-x_{1}-y_{1}-|x-y|}\left(x_{1}-y_{1}\right)^{\gamma_{1}}\left(x^{\prime}-y^{\prime}\right)^{\gamma^{\prime}}|x-y|^{\frac{\alpha_{1}-\widetilde{\alpha}_{1}-\gamma_{1}-\left|y^{\prime}\right|-n-1}{2}}\left(1+O\left(|x-y|^{-1}\right)\right),
$$

whose modulus is controlled by

$$
e^{-x_{1}-y_{1}-|x-y|}|x-y|^{\frac{\alpha_{1}-\left(\widetilde{\alpha}_{1}-\gamma_{1}\right)-n-1}{2}}\left(\frac{\left|x^{\prime}-y^{\prime}\right|^{2}}{|x-y|}\right)^{\frac{\left|y^{\prime}\right|}{2}} .
$$

Now (2.3) follows, since $\alpha_{1} \leq q$ and $\gamma_{1} \leq \widetilde{\alpha}_{1}$ and also $0 \leq\left|\gamma^{\prime}\right| \leq\left|\alpha^{\prime}\right|=k-\alpha_{1}$.

To verify (2.4), assume that $x_{1}-y_{1}>0$ is large and that $\left|x^{\prime}-y^{\prime}\right|^{2} /\left(x_{1}-y_{1}\right)$ stays bounded. In this argument, we will neglect all terms that are much smaller than the right-hand side of (2.4). From (2.11) and (2.12), we then see that in (2.9) we need only take $\alpha_{1}=q$, which implies $\left|\alpha^{\prime}\right|=k-q$, and $\gamma_{1}=\widetilde{\alpha}_{1}$. The latter equality means that in the Hermite polynomial $H_{\widetilde{\alpha}_{1}}$ in (2.9), we only consider the leading term, which is

$$
2^{\widetilde{\alpha}_{1}}\left(\frac{x_{1}-y_{1}}{2 \sqrt{t}}\right)^{\widetilde{\alpha}_{1}}=t^{-\frac{\widetilde{\alpha}_{1}}{2}}\left(x_{1}-y_{1}\right)^{\widetilde{\alpha}_{1}} \text {. }
$$

Instead of (2.9), we will now have

$$
(-1)^{k} 2^{-|\widetilde{\alpha}|} e^{-x_{1}-y_{1}}\left(x_{1}-y_{1}\right)^{\widetilde{\alpha}_{1}} \int_{0}^{+\infty} t^{\frac{q-2 \widetilde{\alpha}_{1}-n}{2}} H_{\alpha^{\prime}}\left(\frac{x^{\prime}-y^{\prime}}{2 \sqrt{t}}\right) e^{-t} e^{-\frac{|x-y|^{2}}{4 t}} \frac{d t}{t} .
$$

Applying (2.5) to the integral here, we see that (2.9) amounts to a positive constant times

$$
\begin{aligned}
& (-1)^{k} 2^{-|\widetilde{\alpha}|} e^{-x_{1}-y_{1}-|x-y|}\left(x_{1}-y_{1}\right)^{\widetilde{\alpha}_{1}}\left(\frac{|x-y|}{2}\right)^{\frac{q-2 \widetilde{\alpha}_{1}-n}{2}} \\
& \times|x-y|^{-\frac{1}{2}} H_{\alpha^{\prime}}\left(\frac{x^{\prime}-y^{\prime}}{\sqrt{2}|x-y|^{1 / 2}}\right)\left(1+O\left(|x-y|^{-1}\right)\right) .
\end{aligned}
$$


Here, we can replace the powers of $|x-y|$ by the same powers of $x_{1}-y_{1}$, since $|x-y|=$ $x_{1}-y_{1}+O(1)$, so (2.13) equals

$$
\begin{aligned}
& (-1)^{k} 2^{-\frac{q}{2}+\widetilde{\alpha}_{1}-|\widetilde{\alpha}|+\frac{n}{2}} e^{-x_{1}-y_{1}-|x-y|}\left(x_{1}-y_{1}\right)^{\frac{q-n-1}{2}} \\
& \quad \times H_{\alpha^{\prime}}\left(\frac{x^{\prime}-y^{\prime}}{\sqrt{2}\left(x_{1}-y_{1}\right)^{1 / 2}}\right)\left(1+O\left(|x-y|^{-1}\right)\right) .
\end{aligned}
$$

Summing over $0 \leq \widetilde{\alpha}_{1} \leq q$, we conclude that

$$
\begin{aligned}
\partial_{x}^{\alpha}\left(-\Delta_{e_{1}}\right)^{-k / 2}(x, y)= & (-1)^{k} b_{\alpha} e^{-x_{1}-y_{1}-|x-y|}\left(x_{1}-y_{1}\right)^{\frac{q-n-1}{2}} \\
& \times H_{\alpha^{\prime}}\left(\frac{x^{\prime}-y^{\prime}}{\sqrt{2}\left(x_{1}-y_{1}\right)^{1 / 2}}\right)\left(1+O\left(|x-y|^{-1}\right)\right)
\end{aligned}
$$

for some $b_{\alpha}>0$. Here, $\alpha=\left(q, \alpha^{\prime}\right)$ and $|\alpha|=k$, and if we sum over such $\alpha$ with the coefficients from (1.1), the result will be

$$
\begin{aligned}
R_{D}(x, y)= & (-1)^{k} e^{-x_{1}-y_{1}-|x-y|}\left(x_{1}-y_{1}\right)^{\frac{q-n-1}{2}} \sum a_{\alpha} b_{\alpha} H_{\alpha^{\prime}}\left(\frac{x^{\prime}-y^{\prime}}{\sqrt{2}\left(x_{1}-y_{1}\right)^{1 / 2}}\right) \\
& + \text { negligible terms. }
\end{aligned}
$$

Since the $a_{\alpha}$ do not all vanish, the orthogonality property of the Hermite polynomials implies that the polynomial given by the sum here is not identically 0 . To finish the proof of (2.4), we need only take a closed ball $B$ where this polynomial does not vanish.

\section{Proof of Theorem 1.1}

We split $R_{D}$ into a part at infinity

$$
R_{D}^{\infty} f(x)=\int_{|x-y|>1} R_{D}(x, y) f(y) d \mu(y)
$$

and a local part $R_{D}^{\text {loc }}=R_{D}-R_{D}^{\infty}$.

The local part is easy to treat. Because of the local doubling property, we can use the method of localization. In view of Proposition 2.1, standard Calderón-Zygmund singular integral theory gives the weak type $(1,1)$ of $R_{D}^{\text {loc }}$.

To estimate $R_{D}^{\infty}$, we start with that part defined by the restriction $x_{1}-y_{1} \leq 1$. Then we have integrability, since we get, using (2.3) and replacing $d \mu(x)$ by $e^{2 x_{1}} d x$,

$$
\begin{aligned}
& \int_{\substack{|x-y|>1 \\
x_{1}-y_{1} \leq 1}}\left|R_{D}(x, y)\right| d \mu(x) \\
& \quad \lesssim \int_{|x-y|>1} e^{1-|x-y|}|x-y|^{\frac{q-n-1}{2}}\left[1+\left(\frac{\left|x^{\prime}-y^{\prime}\right|^{2}}{|x-y|}\right)^{\frac{k}{2}}\right] d x \\
& \quad \lesssim 1,
\end{aligned}
$$

uniformly in $y$. It follows that this part of the operator is of strong type $(1,1)$. 
To treat the opposite case $x_{1}-y_{1}>1$, we write the first exponent in (2.3) as

$$
-2 x_{1}-\left(|x-y|-\left(x_{1}-y_{1}\right)\right)
$$

and observe that in this case,

$$
|x-y|-\left(x_{1}-y_{1}\right)=\frac{\left|x^{\prime}-y^{\prime}\right|^{2}}{|x-y|+x_{1}-y_{1}}
$$

and

$$
\frac{\left|x^{\prime}-y^{\prime}\right|^{2}}{2|x-y|} \leq \frac{\left|x^{\prime}-y^{\prime}\right|^{2}}{|x-y|+x_{1}-y_{1}} \leq \frac{\left|x^{\prime}-y^{\prime}\right|^{2}}{|x-y|} .
$$

Thus, Proposition 2.2 implies that for $x_{1}-y_{1}>1$,

$$
\left|R_{D}(x, y)\right| \lesssim e^{-2 x_{1}}|x-y|^{\frac{q-n-1}{2}} \exp \left(-\frac{\left|x^{\prime}-y^{\prime}\right|^{2}}{2|x-y|}\right)\left[1+\left(\frac{\left|x^{\prime}-y^{\prime}\right|^{2}}{|x-y|}\right)^{\frac{k}{2}}\right] .
$$

It follows that this part of $\left|R_{D}(x, y)\right|$ is controlled by the kernel

$$
\mathcal{V}_{\kappa}(x, y)=e^{-2 x_{1}}|x-y|^{\frac{\kappa-n-1}{2}} \exp \left(-\frac{1}{4} \frac{\left|x^{\prime}-y^{\prime}\right|^{2}}{|x-y|}\right) \chi_{\left\{x_{1}-y_{1}>1\right\}},
$$

with $\kappa=q$.

For $\kappa \in \mathbb{R}$ and suitable functions $f$, let

$$
\widetilde{\mathcal{V}_{\kappa}} f(x)=\int_{\mathbb{R}^{n}} \mathcal{V}_{\kappa}(x, y) f(y) d \mu(y) .
$$

To prove Theorem 1.1 in the case $q \leq 2$, it is enough to show the following result.

Proposition 3.1 The operator $\widetilde{V_{2}}$ defined by (3.3) is of weak type $(1,1)$.

The case $n=1$ is trivial, since then

$$
\left|\widetilde{\mathcal{V}_{2}} f(x)\right| \leq e^{-2 x}\|f\|_{L^{1}(d \mu)},
$$

which implies the weak type $(1,1)$ of $\widetilde{V_{2}}$.

In what follows, we suppose that $n \geq 2$, so that

$$
\widetilde{\mathcal{V}_{2}} f(x)=e^{-2 x_{1}} \int_{y_{1}<x_{1}-1}|x-y|^{\frac{1-n}{2}} \exp \left(-\frac{1}{4} \frac{\left|x^{\prime}-y^{\prime}\right|^{2}}{|x-y|}\right) f(y) e^{2 y_{1}} d y,
$$

where we can assume $0 \leq f \in L^{1}(d \mu)$. It will be convenient to use $g(y)=f(y) e^{2 y_{1}} \in$ $L^{1}(d y)$ instead of $f$. When $\left|x^{\prime}-y^{\prime}\right| \lesssim \sqrt{x_{1}-y_{1}}$, the exponential in the integrand is essentially 1 . This indicates the most important part of the operator, dealt with in the following proposition.

Proposition 3.2 The operator

$$
T g(x)=e^{-2 x_{1}} \int_{\substack{x^{\prime}-y^{\prime} \mid<\sqrt{x_{1}-y_{1}} \\ y_{1}<x_{1}-1}}\left(x_{1}-y_{1}\right)^{\frac{1-n}{2}} g(y) d y
$$

maps $L^{1}(d y)$ boundedly into $L^{1, \infty}(d \mu)$. 
We first verify that this proposition implies Proposition 3.1. For $j=1,2, \ldots$, let

$$
\begin{aligned}
& T_{j} g(x)=e^{-2 x_{1}} \int_{2^{j-1} \sqrt{x_{1}-y_{1}} \leq\left|x^{\prime}-y^{\prime}\right|<2^{j} \sqrt{x_{1}-y_{1}}}^{\left.y_{1} x_{1}-y_{1}\right)^{\frac{1-n}{2}}}\left(x_{1}\right. \\
& \times \exp \left(-\frac{1}{4} \frac{\left|x^{\prime}-y^{\prime}\right|^{2}}{|x-y|}\right) g(y) d y ;
\end{aligned}
$$

then $\widetilde{\nu_{2}} f \leq T g+\sum_{1}^{\infty} T_{j} g$. In this integral, the exponential is less than $\exp \left(-c 2^{j}\right)$ for some $c>0$, so that

$$
T_{j} g(x) \leq \exp \left(-c 2^{j}\right) e^{-2 x_{1}} \int_{\substack{x^{\prime}-y^{\prime} \mid<2^{j} \sqrt{x_{1}-y_{1}} \\ y_{1}<x_{1}-1}}\left(x_{1}-y_{1}\right)^{\frac{1-n}{2}} g(y) d y .
$$

Scaling the variables $x^{\prime}$ and $y^{\prime}$ by a factor $2^{j}$, we can control this expression in terms of the operator $T$, still with a rapidly decreasing coefficient. One can then apply Proposition 3.2 and use [30, Lemma 2.3] to sum in weak $L^{1}$. The result will be the weak type $(1,1)$ of $\widetilde{V_{2}}$, which is Proposition 3.1.

Proof of Proposition 3.2 We cover $\mathbb{R}^{n-1}$ and $\mathbb{R}^{n}$ with lattices of unit cubes

$$
Q_{m}=\left\{x^{\prime}=\left(x_{2}, \ldots, x_{n}\right) \in \mathbb{R}^{n-1}: m_{v}-1<x_{v} \leq m_{v}, \quad v=2, \ldots, n\right\},
$$

where $m=\left(m_{2}, \ldots, m_{n}\right) \in \mathbb{Z}^{n-1}$, and

$$
Q_{i, m}=(i-1, i] \times Q_{m}, \quad(i, m) \in \mathbb{Z} \times \mathbb{Z}^{n-1} .
$$

In this proof, the word "cube" will always refer to cubes of these lattices.

Given $0 \leq g \in L^{1}(d y)$ and $\lambda>0$, we must estimate the $\mu$ measure of the level set $L_{\lambda}=\{x: \operatorname{Tg}(x)>\lambda\}$. Since $\operatorname{Tg}(x) \leq e^{-2 x_{1}} \int g(y) d y$, the coordinate $x_{1}$ is bounded above on $L_{\lambda}$. Since a translation of $g$ in the $x_{1}$ direction does not change the quasinorm of $T g$ in $L^{1, \infty}(d \mu)$, we can, without restriction, assume that $L_{\lambda}$ is contained in the left half-plane $\left\{x: x_{1} \leq 0\right\}$.

The aim of the following lemma is to discretize the variable $x_{1}$.

Lemma 3.3 Let $i \leq 0$ and assume that the cube $Q_{i, m}$ intersects the level set $L_{\lambda}$. Then $\widetilde{T} g\left(i, x^{\prime}\right)>e^{-2} 2^{-(n-1) / 2} \lambda$ for all $x^{\prime} \in Q_{m}$, where

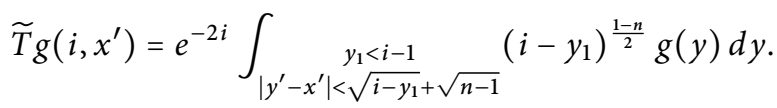

Proof Take a point $z \in Q_{i, m} \cap L_{\lambda}$, so that

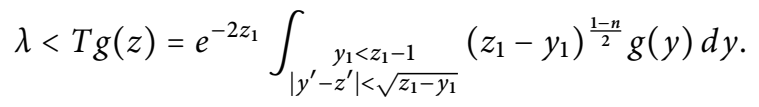

Here, $i<1+z_{1}$ and $z_{1}-y_{1}>1$, so that $i-y_{1}<1+z_{1}-y_{1}<2\left(z_{1}-y_{1}\right)$, and thus $\left(z_{1}-y_{1}\right)^{(1-n) / 2}<2^{(n-1) / 2}\left(i-y_{1}\right)^{(1-n) / 2}$. Further, the region of integration in (3.6) is contained in that of (3.5), because $z_{1} \leq i$ and $\left|y^{\prime}-x^{\prime}\right| \leq\left|y^{\prime}-z^{\prime}\right|+\left|z^{\prime}-x^{\prime}\right| \leq \mid y^{\prime}-$ $z^{\prime} \mid+\sqrt{n-1}$ for any $x^{\prime} \in Q_{m}$. Since also $e^{-2 z_{1}} \leq e^{2} e^{-2 i}$, it follows that $\operatorname{Tg}(z)<$ $e^{2} 2^{(n-1) / 2} \widetilde{T} g\left(i, x^{\prime}\right)$, which proves the lemma. 
The region of integration in (3.5) is contained in the set

$$
D_{i, m}=\bigcup_{x^{\prime} \in Q_{m}}\left\{\left(y_{1}, y^{\prime}\right): y_{1}<i-1,\left|y^{\prime}-x^{\prime}\right|<\sqrt{i-y_{1}}+\sqrt{n-1}\right\} .
$$

For each cube $Q_{i, m}$, we define a family of "forbidden" cubes

$$
F_{i, m}=\left\{Q_{i^{\prime}, m^{\prime}}: i^{\prime}<i,\left|m-m^{\prime}\right|<4 \sqrt{i-i^{\prime}}+4 \sqrt{n-1}\right\}
$$

and their union

$$
\widehat{F}_{i, m}=\bigcup_{Q \in F_{i, m}} Q .
$$

The exponential behavior of $\mu$ implies that

$$
\mu\left(\widehat{F}_{i, m}\right) \lesssim \mu\left(Q_{i, m}\right) .
$$

For each $i \leq 0$, we will select some of the cubes $Q_{i, m}$, namely those for which $m$ is in a set $A_{i} \subset \mathbb{Z}^{n-1}$. These $A_{i}$ will be defined by recursion. We will have the inclusion

$$
L_{\lambda} \subset \bigcup_{i \leq 0} \bigcup_{m \in A_{i}}\left(Q_{i, m} \cup \widehat{F}_{i, m}\right) .
$$

Because of (3.7), this implies that

$$
\mu\left(L_{\lambda}\right) \lesssim \sum_{i \leq 0} \sum_{m \in A_{i}} \mu\left(Q_{i, m}\right) \sim \sum_{i \leq 0} \sum_{m \in A_{i}} e^{2 i} .
$$

When we make the selection, i.e., define the $A_{i}$, we will consider the $\widehat{F}_{i, m}$ as forbidden regions, where no selection is allowed. Indeed, we will select for each $i=0,-1,-2, \ldots$, those cubes $Q_{i, m}$ that intersect the level set and are not in $F_{j, \ell}$ for any already selected cube $Q_{j, \ell}$. In this way, cubes selected at different steps will be far from each other.

More precisely, we first let

$$
A_{0}=\left\{m \in \mathbb{Z}^{n-1}: Q_{0, m} \cap L_{\lambda} \neq \emptyset\right\} .
$$

Assuming for some $i<0$, the sets $A_{i+1}, \ldots, A_{0}$ are already defined, we then let

$$
A_{i}=\left\{m \in \mathbb{Z}^{n-1}: Q_{i, m} \cap L_{\lambda} \neq \emptyset \text { and } Q_{i, m} \notin F_{j, \ell} \text { for all } j>i, \ell \in A_{j}\right\} .
$$

The inclusion (3.8) is immediate from this construction.

For each $i \leq 0$, we define the set

$$
D_{i}=\bigcup_{m \in A_{i}} D_{i, m}
$$

which is contained in $\left\{y: y_{1} \leq i-1\right\}$. In order to replace the $D_{i}$ by pairwise disjoint sets, we let $E_{0}=D_{0}$ and

$$
E_{i}=D_{i} \backslash \bigcup_{j=i+1}^{0} D_{j}, \quad i=-1,-2, \ldots
$$


Then we let $g_{i}=g \chi_{E_{i}}$ and observe that $g=\sum_{j=i}^{0} g_{j}$ in $D_{i}$. This together with Lemma 3.3 implies that, for $i \leq 0$ and $x^{\prime} \in Q_{m}$ with $m \in A_{i}$,

$$
\lambda \lesssim \widetilde{T} g\left(i, x^{\prime}\right)=e^{-2 i} \sum_{j=i}^{0} \int_{y_{1}<i-1}\left(i-y_{1}\right)^{\frac{1-n}{2}} \int_{\left|y^{\prime}-x^{\prime}\right|<\sqrt{i-y_{1}}+\sqrt{n-1}} g_{j}(y) d y^{\prime} d y_{1} .
$$

The integral in $y^{\prime}$ in this expression can be seen as a convolution in $\mathbb{R}^{n-1}$. That leads to an estimate that is insufficient for our purpose, essentially because several values of $i$ are used for the same $g_{j}$. To do better, we will replace $i$ by $j$ in the iterated integral in (3.10). Thus, we first claim that

$$
\left(i-y_{1}\right)^{\frac{1-n}{2}} g_{j}(y) \lesssim\left(j-y_{1}\right)^{\frac{1-n}{2}} g_{j}(y)
$$

if $y, x^{\prime}, m, i$, and $j$ are as in (3.10). Then we observe that an immediate consequence of (3.10) and (3.11) is that if $x^{\prime} \in Q_{m}$ for some $m \in A_{i}$, then

$$
\lambda \lesssim e^{-2 i} \sum_{j=i}^{0} \int_{y_{1}<j-1}\left(j-y_{1}\right)^{\frac{1-n}{2}} \int_{\left|y^{\prime}-x^{\prime}\right|<\sqrt{j-y_{1}}+\sqrt{n-1}} g_{j}(y) d y^{\prime} d y_{1},
$$

where we also made the regions of integration larger.

Before we use (3.12) to finish the proof of Proposition 3.2, we verify claim (3.11). Then we can obviously assume that $i<j$ and $g_{j}(y) \neq 0$, which implies $y \in E_{j} \subset D_{j}$. The definition of $D_{j}$ says that $y_{1}<j-1$ and $\left|y^{\prime}-z^{\prime}\right|<\sqrt{j-y_{1}}+\sqrt{n-1}$ for some $z^{\prime} \in Q_{\ell}$ and some $\ell \in A_{j}$. Clearly, $\left|z^{\prime}-\ell\right| \leq \sqrt{n-1}$, so the triangle inequality leads to

$$
\left|y^{\prime}-\ell\right|<\sqrt{j-y_{1}}+2 \sqrt{n-1}
$$

The inner integral in (3.10) is taken over those $y^{\prime}$ satisfying

$$
\left|y^{\prime}-x^{\prime}\right|<\sqrt{i-y_{1}}+\sqrt{n-1} .
$$

Since also $\left|x^{\prime}-m\right| \leq \sqrt{n-1},(3.13)$ and (3.14) imply

$$
|m-\ell|<\sqrt{j-y_{1}}+\sqrt{i-y_{1}}+4 \sqrt{n-1} \leq 2 \sqrt{j-y_{1}}+4 \sqrt{n-1} .
$$

On the other hand, the cube $Q_{i, m}$ was selected and thus not forbidden by the selected cube $Q_{j, \ell}$, i.e., $Q_{i, m} \notin F_{j, \ell}$. This means that $|m-\ell| \geq 4 \sqrt{j-i}+4 \sqrt{n-1}$. Combining this inequality with (3.15), we get $4 \sqrt{j-i}<2 \sqrt{j-y_{1}}$, so that $j-i<$ $\left(j-y_{1}\right) / 4$. Then we can write

$$
j-y_{1}=j-i+i-y_{1}<\frac{1}{4}\left(j-y_{1}\right)+i-y_{1}
$$

and thus

$$
j-y_{1}<\frac{4}{3}\left(i-y_{1}\right)
$$

This implies (3.11), and (3.12) also follows.

We now integrate inequality (3.12) in $x^{\prime}$ over the unit cube $Q_{m}$, to conclude that

$$
e^{2 i} \lesssim \frac{1}{\lambda} \sum_{j=i}^{0} \int_{y_{1}<j-1} \int_{Q_{m}}\left(j-y_{1}\right)^{\frac{1-n}{2}} \int_{\left|y^{\prime}-x^{\prime}\right|<\sqrt{j-y_{1}}+\sqrt{n-1}} g_{j}(y) d y^{\prime} d x^{\prime} d y_{1} .
$$


The inner part of this expression,

$$
G_{j}\left(y_{1}, x^{\prime}\right):=\left(j-y_{1}\right)^{\frac{1-n}{2}} \int_{\left|y^{\prime}-x^{\prime}\right|<\sqrt{j-y_{1}}+\sqrt{n-1}} g_{j}\left(y_{1}, y^{\prime}\right) d y^{\prime},
$$

is the value at $x^{\prime}$ of the convolution in $\mathbb{R}^{n-1}$ of $g_{j}\left(y_{1}, \cdot\right)$ and the characteristic function of the ball $\left\{y^{\prime} \in \mathbb{R}^{n-1}:\left|y^{\prime}\right|<\sqrt{j-y_{1}}+\sqrt{n-1}\right\}$, essentially normalized in $L^{1}\left(\mathbb{R}^{n-1}\right)$ by the first factor. Thus,

$$
\int_{\mathbb{R}^{n-1}} G_{j}\left(y_{1}, x^{\prime}\right) d x^{\prime} \lesssim \int_{\mathbb{R}^{n-1}} g_{j}\left(y_{1}, y^{\prime}\right) d y^{\prime},
$$

uniformly in $j-y_{1}>1$.

Combining (3.16) with (3.9), we have

$$
\begin{aligned}
\mu\left(L_{\lambda}\right) & \lesssim \frac{1}{\lambda} \sum_{i \leq 0} \sum_{m \in A_{i}} \sum_{j=i}^{0} \int_{y_{1}<j-1} \int_{Q_{m}} G_{j}\left(y_{1}, x^{\prime}\right) d x^{\prime} d y_{1} \\
& =\frac{1}{\lambda} \sum_{j \leq 0} \int_{y_{1}<j-1} \sum_{i=-\infty}^{j} \sum_{m \in A_{i}} \int_{Q_{m}} G_{j}\left(y_{1}, x^{\prime}\right) d x^{\prime} d y_{1} .
\end{aligned}
$$

In the last expression here, we have a double sum of integrals over $Q_{m}$, taken over all $m \in A_{i}$ and $i=j, j-1, \ldots$. The corresponding family of cubes $Q_{m}$ is pairwise disjoint, since $m \in A_{i}$ implies $Q_{i^{\prime}, m} \in F_{i, m}$ for $i^{\prime}<i$ by the definition of $F_{i, m}$. Thus, the double sum of integrals over $Q_{m}$ is no larger than the left-hand side of (3.17). As a result,

$$
\begin{aligned}
\mu\left(L_{\lambda}\right) & \lesssim \frac{1}{\lambda} \sum_{j \leq 0} \int_{y_{1}<j-1} \int_{\mathbb{R}^{n-1}} g_{j}\left(y_{1}, y^{\prime}\right) d y^{\prime} d y_{1} \\
& \leq \frac{1}{\lambda} \sum_{j \leq 0} \int_{\mathbb{R}^{n}} g_{j}(y) d y \leq \frac{1}{\lambda} \int g(y) d y .
\end{aligned}
$$

This ends the proof of Proposition 3.2.

Now consider the case $q>2$ in Theorem 1.1. The main part of the kernel $R_{D}(x, y)$ is controlled by the kernel $\mathcal{V}_{q}$ defined in (3.2). Therefore, the estimate (1.2) is a consequence of the following proposition.

Proposition 3.4 For any $\kappa>2$, there exists a constant $C=C(\kappa, n)>0$ such that for all $f \in L\left(1+\ln ^{+} L\right)^{\kappa / 2-1}(\mu)$ and $\lambda>0$, we have

$$
\mu\left\{x ;\left|\widetilde{V_{\kappa}} f(x)\right|>\lambda\right\} \leq C \int \frac{|f|}{\lambda}\left(1+\ln ^{+} \frac{|f|}{\lambda}\right)^{\frac{\kappa}{2}-1} d \mu,
$$

where the operator $\widetilde{V_{\kappa}}$ is defined by (3.3).

Proof For $h \in L^{1}(\mu)$, we have

$$
\widetilde{\mathcal{V}_{\kappa}} h(x)=\int_{y_{1}<x_{1}-1} e^{-2\left(x_{1}-y_{1}\right)}|x-y|^{\frac{\kappa-n-1}{2}} \exp \left(-\frac{1}{4} \frac{\left|x^{\prime}-y^{\prime}\right|^{2}}{|x-y|}\right) h(y) d y .
$$

Here, we assume $h \geq 0$ and apply the elementary inequality

$$
a b^{\frac{\kappa}{2}-1} \leq C_{0}\left[a\left(1+\ln ^{+} a\right)^{\frac{\kappa}{2}-1}+e^{\frac{b}{8}}\right], \quad a, b>0,
$$


easily proved by separating the cases $b \leq 10\left(1+\ln ^{+} a\right)$ and $b>10\left(1+\ln ^{+} a\right)$. The constant $C_{0}$ depends only on $\kappa$. Letting $a=h(y)$ and $b=|x-y|$, we get

$$
\begin{aligned}
\widetilde{\mathcal{V}_{\kappa}} h(x) \leq & C_{0} \widetilde{\mathcal{V}_{2}}\left(h\left(1+\ln ^{+} h\right)^{\frac{\kappa}{2}-1}\right)(x) \\
& +C_{0} \int_{y_{1}<x_{1}-1} e^{-2\left(x_{1}-y_{1}\right)}|x-y|^{\frac{1-n}{2}} \exp \left(-\frac{1}{4} \frac{\left|x^{\prime}-y^{\prime}\right|^{2}}{|x-y|}\right) e^{\frac{|x-y|}{8}} d y .
\end{aligned}
$$

The integral here equals

$$
\int_{z_{1}>1} e^{-2 z_{1}}|z|^{\frac{1-n}{2}} \exp \left(-\frac{1}{4} \frac{\left|z^{\prime}\right|^{2}}{|z|}\right) e^{\frac{|z|}{8}} d z,
$$

and to see that it is finite, we split it in two:

$$
\int_{\left|z^{\prime}\right|<2 z_{1}}^{z_{1}>1}+\int_{\left|z^{\prime}\right|>2 z_{1}}=I_{1}+I_{2}
$$

In $I_{1}$, we can estimate the second and third exponential factors by 1 and $e^{3 z_{1} / 8}$, respectively, and integrate first in $z^{\prime}$. In $I_{2}$, we have $|z|<3\left|z^{\prime}\right| / 2$ and thus $\left|z^{\prime}\right|^{2} /|z|>$ $2\left|z^{\prime}\right| / 3$, so that the second exponential in $I_{2}$ is less than $\exp \left(-\left|z^{\prime}\right| / 6\right)$. It is then enough to estimate the third exponential by $\exp \left(z_{1} / 8+\left|z^{\prime}\right| / 8\right)$.

It now follows that

$$
\widetilde{V_{\kappa}} h(x) \leq C_{0} \widetilde{V_{2}}\left(h\left(1+\ln ^{+} h\right)^{\frac{\kappa}{2}-1}\right)(x)+C_{1}
$$

for some constant $C_{1}$.

Given $f \in L\left(1+\ln ^{+} L\right)^{(\kappa-2) / 2}(\mu)$ and $\lambda>0$, we let $h=2 C_{1} \lambda^{-1}|f|$. Then $\left|\widetilde{\mathcal{V}_{\kappa}} f(x)\right|>$ $\lambda$ implies $\widetilde{V_{\kappa}}(h)(x)>2 C_{1}$ and $\widetilde{V_{2}}\left(h\left(1+\ln ^{+} h\right)^{(\kappa-2) / 2}\right)(x)>C_{1} / C_{0}$ because of (3.19). Since $\widetilde{V_{2}}$ is of weak type $(1,1)$ by Proposition 3.1, we conclude that

$$
\mu\left\{\widetilde{\mathcal{V}_{\kappa}} f(x) \mid>\lambda\right\} \lesssim\left\|h\left(1+\ln ^{+} h\right)^{\frac{\kappa}{2}-1}\right\|_{L^{1}(\mu)} \sim \int \frac{|f|}{\lambda}\left(1+\ln ^{+} \frac{|f|}{\lambda}\right)^{\frac{\kappa}{2}-1} d \mu .
$$

Proposition 3.4 is proved.

To show that (1.2) is sharp, we will use (2.4).

Let $f=\chi_{B(0,1)}$. With $\eta>0$ large, we consider $R_{D} f(x)$ in the region

$$
\Omega_{\eta}=\left\{x: \eta<x_{1}<\eta+1, \quad \frac{x^{\prime}}{\sqrt{\eta}} \in \frac{1}{2} B\right\} .
$$

Here, $\frac{1}{2} B$ denotes the concentric scaling of the ball $B$ from (2.4), by a factor $1 / 2$. Let $x \in \Omega_{\eta}$ and $y \in B(0,1)$. Then

$$
\frac{x^{\prime}-y^{\prime}}{\left(x_{1}-y_{1}\right)^{1 / 2}}=\frac{x^{\prime}}{\sqrt{\eta}}+O\left(\frac{1}{\sqrt{\eta}}\right), \quad \eta \rightarrow \infty .
$$

This implies that $\left(x^{\prime}-y^{\prime}\right) /\left(x_{1}-y_{1}\right)^{1 / 2} \in B$ for large $\eta$, and (2.4) applies. Further, (3.1) shows that $e^{-x_{1}-y_{1}-|x-y|} \sim e^{-2 x_{1}}$, and so

$$
(-1)^{k} R_{D} f(x) \sim e^{-2 \eta} \eta^{\frac{q-n-1}{2}}=\lambda
$$


for $x \in \Omega_{\eta}$, where the equality defines $\lambda$. Then $\eta \sim \ln \lambda^{-1}$, and

$$
\mu\left(\Omega_{\eta}\right) \sim e^{2 \eta} \eta^{\frac{n-1}{2}}=\lambda^{-1} \eta^{\frac{q-n-1}{2}} \eta^{\frac{n-1}{2}} \sim \lambda^{-1}\left(\ln \lambda^{-1}\right)^{\frac{q}{2}-1} .
$$

For $q>2$ this shows that (1.2) is sharp, and in particular that $R_{D}$ is not of weak type $(1,1)$.

Theorem 1.1 is completely proved.

\section{Proof of Theorem 1.2(i)}

Before proving this theorem, we find local and global estimates for $t^{k / 2} D_{x} p_{t}(x, y)$ in the space $L^{2}(d t / t)$. They will be analogous to Propositions 2.1 and 2.2, with similar proofs.

Proposition 4.1 For $0<|x-y| \leq 1$, one has

$$
\begin{aligned}
\left\|t^{\frac{k}{2}} D_{x} p_{t}(x, y)\right\|_{L^{2}(d t / t)} & \lesssim \frac{1}{\mu(B(x,|x-y|))}, \\
\left\|t^{\frac{k}{2}} \nabla_{y} D_{x} p_{t}(x, y)\right\|_{L^{2}(d t / t)} & \lesssim \frac{1}{|x-y| \mu(B(x,|x-y|))} .
\end{aligned}
$$

Proposition 4.2 For $|x-y|>1$, one has

$$
\left\|t^{\frac{k}{2}} D_{x} p_{t}(x, y)\right\|_{L^{2}(d t / t)} \lesssim e^{-x_{1}-y_{1}-|x-y|}|x-y|^{\frac{q-n}{2}-\frac{1}{4}}\left[1+\left(\frac{\left|x^{\prime}-y^{\prime}\right|^{2}}{|x-y|}\right)^{\frac{k}{2}}\right] .
$$

Proof of Propositions 4.1 and 4.2 Clearly,

$$
\left|t^{\frac{k}{2}} D_{x} p_{t}(x, y)\right|^{2}=\sum_{|\alpha|=|\beta|=k} a_{\alpha} \overline{a_{\beta}} t^{k} \partial_{x}^{\alpha} p_{t}(x, y) \partial_{x}^{\beta} p_{t}(x, y) .
$$

We write $\alpha=\left(\alpha_{1}, \alpha^{\prime}\right)$ as before, and similarly, $\beta=\left(\beta_{1}, \beta^{\prime}\right)$, assuming always $|\alpha|=|\beta|=$ $k$. Using expression (1.8) for $p_{t}(x, y)$ and (2.8), we see that $t^{k} \partial_{x}^{\alpha} p_{t}(x, y) \partial_{x}^{\beta} p_{t}(x, y)$ is the sum of positive factors times

$$
e^{-2 x_{1}-2 y_{1}} t^{k-\frac{|\widetilde{\alpha}+| \widetilde{\beta} \mid}{2}-n} H_{\widetilde{\alpha}}\left(\frac{x-y}{2 \sqrt{t}}\right) H_{\widetilde{\beta}}\left(\frac{x-y}{2 \sqrt{t}}\right) e^{-2 t} e^{-\frac{|x-y|^{2}}{2 t}},
$$

taken over $\widetilde{\alpha}_{1}=0, \ldots, \alpha_{1}$ and $\widetilde{\beta}_{1}=0, \ldots, \beta_{1}$. Here as in Section 2 , we have $\widetilde{\alpha}=\left(\widetilde{\alpha}_{1}, \alpha^{\prime}\right)$, and similarly $\widetilde{\beta}=\left(\widetilde{\beta}_{1}, \beta^{\prime}\right)$.

Integrating this expression with respect to $d t / t$, we obtain a positive constant times

$$
e^{-2 x_{1}-2 y_{1}} \int_{0}^{+\infty} t^{k-\frac{|\widetilde{\alpha}+| \widetilde{\beta} \mid}{2}-n} H_{\widetilde{\alpha}}\left(\frac{x-y}{2 \sqrt{t}}\right) H_{\widetilde{\beta}}\left(\frac{x-y}{2 \sqrt{t}}\right) e^{-2 t} e^{-\frac{|x-y|^{2}}{2 t}} \frac{d t}{t} .
$$

Here, the product of the two Hermite polynomials is a linear combination of terms

$$
\left(\frac{x_{1}-y_{1}}{2 \sqrt{t}}\right)^{\gamma_{1}}\left(\frac{x^{\prime}-y^{\prime}}{2 \sqrt{t}}\right)^{\gamma^{\prime}}\left(\frac{x_{1}-y_{1}}{2 \sqrt{t}}\right)^{\delta_{1}}\left(\frac{x^{\prime}-y^{\prime}}{2 \sqrt{t}}\right)^{\delta^{\prime}},
$$


where $\gamma=\left(\gamma_{1}, \gamma^{\prime}\right)$ and $\delta=\left(\delta_{1}, \delta^{\prime}\right)$ satisfy $0 \leq \gamma_{1} \leq \widetilde{\alpha}_{1}$ and $0 \leq \gamma^{\prime} \leq \alpha^{\prime}$, and similarly, $0 \leq \delta_{1} \leq \widetilde{\beta}_{1}$ and $0 \leq \delta^{\prime} \leq \beta^{\prime}$. Each such term leads to a corresponding term in the expression (4.4), proportional to

$$
e^{-2 x_{1}-2 y_{1}}\left(x_{1}-y_{1}\right)^{\gamma_{1}+\delta_{1}}\left(x^{\prime}-y^{\prime}\right)^{\gamma^{\prime}+\delta^{\prime}} \int_{0}^{+\infty} t^{k-\frac{|\widetilde{x}|+|\widetilde{\beta}+| y|+| \delta \mid}{2}-n} e^{-2 t} e^{-\frac{|x-y|^{2}}{2 t}} \frac{d t}{t} .
$$

After replacing $t$ by $\tau=2 t$ in this integral, one can apply Lemma 2.3 with $a=2|x-y|$.

For $|x-y| \leq 1$, we see from Lemma 2.3(i) that the modulus of (4.5) is controlled by

$$
\begin{aligned}
& \left.e^{-2 x_{1}-2 y_{1}}\left|x_{1}-y_{1}\right|^{\gamma_{1}+\delta_{1}}\left|x^{\prime}-y^{\prime}\right|\right|^{\prime}|+| \delta^{\prime}|x-y|^{2 k-(|\widetilde{\alpha}|+|\widetilde{\beta}|+|\gamma|+|\delta|)-2 n} \\
& \lesssim e^{-4 x_{1}}|x-y|^{-2 n},
\end{aligned}
$$

if the exponent of $t$ in the integral is negative. But in the opposite case, this is also true. Thus, (4.1) is proved. To complete the proof of Proposition 4.1, one verifies (4.2) in a similar way.

Aiming at Proposition 4.2, we assume that $|x-y|>1$ and conclude from Lemma 2.3(ii) that (4.5) is in modulus bounded by constant times

$$
e^{-2 x_{1}-2 y_{1}-2|x-y|}\left|x_{1}-y_{1}\right|^{\gamma_{1}+\delta_{1}}\left|x^{\prime}-y^{\prime}\right|^{\left|\gamma^{\prime}\right|+\left|\delta^{\prime}\right|}|x-y|^{k-\frac{|\widetilde{\alpha}+| \widetilde{\mid}|+| y|+| \delta \mid}{2}-n-\frac{1}{2}} .
$$

In this expression, we estimate $\left|x_{1}-y_{1}\right|$ by $|x-y|$ and write $k$ as $k=\left(\alpha_{1}+\left|\alpha^{\prime}\right|+\beta_{1}+\right.$ $\left.\left|\beta^{\prime}\right|\right) / 2$, getting

$$
e^{-2 x_{1}-2 y_{1}-2|x-y|}|x-y|^{\frac{\alpha_{1}+\beta_{1}}{2}-\frac{\widetilde{\alpha}_{1}-\gamma_{1}}{2}-\frac{\widetilde{\beta}_{1}-\delta_{1}}{2}-n-\frac{1}{2}}\left(\frac{\left|x^{\prime}-y^{\prime}\right|}{|x-y|^{1 / 2}}\right)^{\left|\gamma^{\prime}\right|+\left|\delta^{\prime}\right|} .
$$

Here, we have

$$
\alpha_{1} \leq q, \quad \beta_{1} \leq q, \quad \widetilde{\alpha}_{1}-\gamma_{1} \geq 0, \quad \widetilde{\beta}_{1}-\delta_{1} \geq 0,
$$

and also $\left|\gamma^{\prime}\right|,\left|\delta^{\prime}\right| \leq k$. Thus, for $|x-y| \geq 1$, the last expression is at most

$$
e^{-2 x_{1}-2 y_{1}-2|x-y|}|x-y|^{q-n-\frac{1}{2}}\left[1+\left(\frac{\left|x^{\prime}-y^{\prime}\right|^{2}}{|x-y|}\right)^{k}\right] \text {. }
$$

We have shown that this is a bound for $\int_{0}^{+\infty} t^{k}\left|D_{x} p_{t}(x, y)\right|^{2} d t / t$, and (4.3) follows. The two propositions are proved.

Remark The estimate at the end of the proof just given can be sharp only when all inequalities (4.7) are equalities. Indeed, if any of the four inequalities is strict, one can estimate (4.6) by a modified quantity (4.8) in which $|x-y|$ has a smaller exponent.

Proof of Theorem 1.2(i) Clearly,

$$
\mathcal{H}_{D}(f)(x)=\left(\int_{0}^{+\infty}\left|t^{\frac{k}{2}} \int D_{x} p_{t}(x, y) f(y) d \mu(y)\right|^{2} \frac{d t}{t}\right)^{\frac{1}{2}} .
$$

We now introduce a local part $\mathcal{H}_{D}^{\text {loc }}$ and a global part $\mathcal{H}_{D}^{\infty}$ of this operator, defined by restricting the integration in $y$ here to the region $|y-x| \leq 1$ and $|y-x|>1$, respectively. 
Thus,

$$
\mathcal{H}_{D} f \leq \mathcal{H}_{D}^{\text {loc }} f+\mathcal{H}_{D}^{\infty} f .
$$

To see that the local part is of weak type $(1,1)$, we apply the method of localization as done for the Riesz transforms, but now using vector-valued singular integral theory and Proposition 4.1.

For $\mathcal{H}_{D}^{\infty}$, we start by applying Minkowski’s integral inequality, getting

$$
\mathcal{H}_{D}^{\infty}(f)(x) \leq \int_{|x-y|>1}|f(y)|\left(\int_{0}^{+\infty}\left|t^{\frac{k}{2}} D_{x} p_{t}(x, y)\right|^{2} \frac{d t}{t}\right)^{\frac{1}{2}} d \mu(y) .
$$

We can now follow the argument for $R_{D}^{\infty}$ in the proof of Theorem 1.1, replacing (2.3) by (4.3). In particular, that part of $\mathcal{H}_{D}^{\infty}$ given by $x_{1}-y_{1} \leq 1$ is seen to be of strong type $(1,1)$. Moreover, the kernel of the remaining part is controlled by $\mathcal{V}_{\kappa}(x, y)$ with $\kappa=q+1 / 2$. This leads to the weak type $(1,1)$ estimate of Theorem 1.2(i) and also to (1.3), in view of Propositions 3.1 and 3.4.

For the sharpness parts of Theorem 1.2(i), we let $f=\chi_{B(0,1)}$. Let $|\alpha|=k$ and take points $y$ and $x$ with $|y|<1$ and $x_{1}>0$ large but $x^{\prime} / \sqrt{|x|}$ bounded, which implies $x_{1}=$ $|x|+O(1)$. We will then estimate $t^{k / 2} \partial_{x}^{\alpha} p_{t}(x, y)$, and we need to be more precise than in the preceding argument. Because of (1.8) and (2.8), this quantity is the sum of

$$
(-1)^{k}\left(\begin{array}{c}
\alpha_{1} \\
\widetilde{\alpha}_{1}
\end{array}\right) 2^{-|\widetilde{\alpha}|} e^{-x_{1}-y_{1}} t^{\frac{k-|\widetilde{\alpha}|-n}{2}} H_{\widetilde{\alpha}}\left(\frac{x-y}{2 \sqrt{t}}\right) e^{-t} e^{-\frac{|x-y|^{2}}{4 t}},
$$

taken over $\widetilde{\alpha}_{1}=0, \ldots, \alpha_{1}$. Here, $\widetilde{\alpha}=\left(\widetilde{\alpha}_{1}, \alpha^{\prime}\right)$ as before. The remark after the end of the proof of Propositions 4.1 and 4.2 shows that we need only consider the case $\alpha_{1}=q$, which implies $\left|\alpha^{\prime}\right|=k-q$. For the same reason, we can replace the polynomial $H_{\widetilde{\alpha}_{1}}\left(\left(x_{1}-y_{1}\right) / 2 \sqrt{t}\right)$ by its leading term $2^{\widetilde{\alpha}_{1}}\left(\left(x_{1}-y_{1}\right) / 2 \sqrt{t}\right)^{\widetilde{\alpha}_{1}}$.

Then we can write

$$
\begin{aligned}
H_{\widetilde{\alpha}}\left(\frac{x-y}{2 \sqrt{t}}\right) & =2^{\widetilde{\alpha}_{1}}\left(\frac{x_{1}-y_{1}}{2 \sqrt{t}}\right)^{\widetilde{\alpha}_{1}} H_{\alpha^{\prime}}\left(\frac{x^{\prime}-y^{\prime}}{2 \sqrt{t}}\right)+\cdots \\
& =2^{\widetilde{\alpha}_{1}} t^{\frac{\widetilde{\alpha}_{1}}{2}}\left(\frac{x_{1}-y_{1}}{2 t}\right)^{\widetilde{\alpha}_{1}} H_{\alpha^{\prime}}\left(\frac{x^{\prime}-y^{\prime}}{2 \sqrt{t}}\right)+\cdots,
\end{aligned}
$$

where the dots indicate negligible terms. Restricting $t$ by $\left|t-\frac{|x|}{2}\right|<|x|^{3 / 4}$, we see that

$$
\begin{aligned}
H_{\widetilde{\alpha}}\left(\frac{x-y}{2 \sqrt{t}}\right) & =2^{\widetilde{\alpha}_{1}} t^{\frac{\widetilde{\alpha}_{1}}{2}}\left(\frac{x_{1}}{|x|}+O\left(|x|^{-\frac{1}{4}}\right)\right)^{\widetilde{\alpha}_{1}} H_{\alpha^{\prime}}\left(\frac{x^{\prime}}{\sqrt{2|x|}}+O\left(|x|^{-\frac{1}{4}}\right)\right)+\cdots \\
& =2^{\widetilde{\alpha}_{1}} t^{\frac{\widetilde{\alpha}_{1}}{2}} H_{\alpha^{\prime}}\left(\frac{x^{\prime}}{\sqrt{2|x|}}\right)+\cdots
\end{aligned}
$$

We now insert this last expression for the Hermite polynomial in (4.9), and observe that $|\widetilde{\alpha}|=\widetilde{\alpha}_{1}+\left|\alpha^{\prime}\right|=\widetilde{\alpha}_{1}+k-q$. Then the only factor in (4.9) that depends on $\widetilde{\alpha}_{1}$ will 
be the binomial coefficient. Since

$$
\sum_{\widetilde{\alpha}_{1}=0}^{\alpha_{1}}\left(\begin{array}{l}
\alpha_{1} \\
\widetilde{\alpha}_{1}
\end{array}\right)=2^{\alpha_{1}}=2^{q}
$$

by summing (4.9) in $\widetilde{\alpha}_{1}$, we get

$$
t^{\frac{k}{2}} \partial_{x}^{\alpha} p_{t}(x, y)=(-1)^{k} 2^{2 q-k} e^{-x_{1}-y_{1}} t^{\frac{q-n}{2}} H_{\alpha^{\prime}}\left(\frac{x^{\prime}}{\sqrt{2|x|}}\right) e^{-t} e^{-\frac{|x-y|^{2}}{4 t}}+\cdots
$$

The next step is to sum these expressions in $\alpha$, with the coefficients from (1.1). The result is that for $y, x$, and $t$ as described above,

$$
\begin{aligned}
t^{\frac{k}{2}} D_{x} & p_{t}(x, y) \\
= & (-1)^{k} 2^{2 q-k} e^{-x_{1}-y_{1}} t^{\frac{q-n}{2}} e^{-t} e^{-\frac{|x-y|^{2}}{4 t}} \sum_{\left|\alpha^{\prime}\right|=k-q} a_{\left(q, \alpha^{\prime}\right)} H_{\alpha^{\prime}}\left(\frac{x^{\prime}}{\sqrt{2|x|}}\right) \\
& + \text { harmless terms. }
\end{aligned}
$$

The sum here is $P\left(x^{\prime} / \sqrt{2|x|}\right)$ for some nonzero polynomial $P$ in $n-1$ variables. Thus, we can find a ball $B \subset \mathbb{R}^{n-1}$ in which $P$ does not vanish. Further,

$$
e^{-\frac{|x-y|^{2}}{4 t}} \sim e^{-\frac{|x|^{2}}{4 t}}
$$

since $|y|<1$ and $t \sim|x|$. With $f=\chi_{B(0,1)}$, by integrating in $y$, we then get

$$
\left|t^{\frac{k}{2}} D e^{t \Delta_{e_{1}}} f(x)\right|^{2} \sim e^{-2 x_{1}} x_{1}^{q-n} e^{-2 t} e^{-\frac{|x|^{2}}{2 t}},
$$

where, as before, $x_{1}>0$ is large, $x^{\prime} / \sqrt{2|x|} \in B$, and $\left|t-\frac{|x|}{2}\right|<|x|^{3 / 4}$. Hence,

$$
\int_{0}^{+\infty}\left|t^{\frac{k}{2}} D e^{t \Delta_{e_{1}}} f(x)\right|^{2} \frac{d t}{t} \gtrsim e^{-2 x_{1}} x_{1}^{q-n} \int_{\frac{|x|}{2}-|x|^{3 / 4}}^{\frac{|x|}{2}+|x|^{3 / 4}} e^{-2 t} e^{-\frac{|x|^{2}}{2 t}} \frac{d t}{t} .
$$

After the change of variable $\tau=2 t$, we can apply the last part of Lemma 2.3(ii) to conclude that the right-hand side of (4.10) equals a positive constant times

$$
e^{-2 x_{1}} x_{1}^{q-n} e^{-2|x|}|x|^{-\frac{1}{2}}\left(1+O\left(|x|^{-1}\right)\right)=e^{-2 x_{1}-2|x|} x_{1}^{q-n-\frac{1}{2}}\left(1+O\left(|x|^{-1}\right)\right) .
$$

We define $\Omega_{\eta}$ by (3.20), though $B$ is not the same as in Section 3. If $\eta$ is large, $x \in \Omega_{\eta}$ implies $x^{\prime} / \sqrt{2|x|} \in B$. It now follows from (4.10) and (4.11) that for large $\eta$,

$$
\mathcal{H}_{D}^{\infty} f \gtrsim e^{-2 \eta} \eta^{\frac{q-n}{2}-\frac{1}{4}}
$$

in $\Omega_{\eta}$. This implies the "only if" part of Theorem 1.2(i) and the sharpness of $q$; $c f$. the very last part of Section 3 . We leave it to the reader to check that all the neglected terms above can be disregarded.

Theorem 1.2(i) is proved. 


\section{Proof of Theorem 1.2(ii)}

To prove the claimed boundedness properties of $\mathcal{G}_{\alpha}$, we copy the reasoning used for Theorems 1.1 and 1.2(i). We must estimate the kernel $t^{k} D_{x} P_{t}(x, y)$ in the space $L^{2}(d t / t)$.

The subordination formula says that the Poisson kernel $P_{t}$ is given by

$$
P_{t}(x, y)=\frac{t}{2 \sqrt{\pi}} \int_{0}^{+\infty} u^{-\frac{3}{2}} e^{-\frac{t^{2}}{4 u}} p_{u}(x, y) d u .
$$

This equation will also hold with $P_{t}(x, y)$ and $p_{u}(x, y)$ replaced by their derivatives $D_{x} P_{t}(x, y)$ and $D_{x} p_{u}(x, y)$, respectively. Using Minkowski's integral inequality, we conclude that

$$
\begin{aligned}
& \left(\int_{0}^{+\infty}\left|t^{k} D_{x} P_{t}(x, y)\right|^{2} \frac{d t}{t}\right)^{\frac{1}{2}} \\
& \quad \sim\left(\int_{0}^{+\infty}\left|t^{k+1} \int_{0}^{+\infty} u^{-\frac{3}{2}} e^{-\frac{t^{2}}{4 u}} D_{x} p_{u}(x, y) d u\right|^{2} \frac{d t}{t}\right)^{\frac{1}{2}} \\
& \quad \leq \int_{0}^{+\infty} u^{-\frac{3}{2}}\left|D_{x} p_{u}(x, y)\right|\left(\int_{0}^{+\infty}\left(t^{k+1} e^{-\frac{t^{2}}{4 u}}\right)^{2} \frac{d t}{t}\right)^{\frac{1}{2}} d u .
\end{aligned}
$$

The last inner integral here is seen to equal $2^{k} \Gamma(k+1) u^{k+1}$, and so

$$
\left(\int_{0}^{+\infty}\left|t^{k} D_{x} P_{t}(x, y)\right|^{2} \frac{d t}{t}\right)^{\frac{1}{2}} \lesssim \int_{0}^{+\infty} u^{\frac{k}{2}}\left|D_{x} p_{u}(x, y)\right| \frac{d u}{u} .
$$

The right-hand side in (5.1) is like the expression for $R_{D}(x, y)$ in (2.7), except for the modulus signs. In Section 2, equality (2.7) was used to prove Propositions 2.1 and 2.2. Tracing those arguments, one sees that inequality (5.1) is sufficient to imply estimates similar to (2.1) and (2.3) for $\left(\int_{0}^{+\infty}\left|t^{k} D_{x} P_{t}(x, y)\right|^{2} d t / t\right)^{1 / 2}$. Taking a derivative in $y$, one also obtains the analog of (2.2). This leads to the weak type of $\mathcal{G}_{\alpha}$ for $q \leq 2$ and also to (1.4), as seen from the proof of Theorem 1.1 in Section 3.

It remains to verify the sharpness parts of Theorem 1.2(ii). Recall that $D=$ $\sum_{|\alpha|=k} a_{\alpha} \partial^{\alpha}$. We will find an $f \in L^{1}(\mu)$ and a ball $B \subset \mathbb{R}^{n-1}$ such that if $x_{1}>0$ is large and $x^{\prime} / \sqrt{x_{1}} \in B$, one has

$$
\mathcal{G}_{D} f(x) \gtrsim e^{-2 x_{1}} x_{1}^{\frac{q-n-1}{2}} .
$$

From this estimate, the sharpness parts of Theorem 1.2(ii) will follow; $c f$. the last few lines of Section 3.

To estimate $D_{x} P_{t}(x, y)$, we consider

$$
\partial_{x}^{\alpha} P_{t}(x, y)=\frac{t}{2 \sqrt{\pi}} \int_{0}^{+\infty} u^{-\frac{3}{2}} e^{-\frac{t^{2}}{4 u}} \partial_{x}^{\alpha} p_{u}(x, y) d u
$$

with $|\alpha|=k$ and $\alpha_{1} \leq q$.

From (1.8), we see that $\partial_{x}^{\alpha} P_{t}(x, y)$ is a sum of terms

$$
(-1)^{\alpha_{1}-\widetilde{\alpha}_{1}}(4 \pi)^{-\frac{n+1}{2}}\left(\begin{array}{l}
\alpha_{1} \\
\widetilde{\alpha}_{1}
\end{array}\right) e^{-x_{1}-y_{1}} t \int_{0}^{+\infty} u^{-\frac{1+n}{2}} e^{-\frac{t^{2}}{4 u}} e^{-u} \partial_{x}^{\widetilde{\alpha}} e^{-\frac{|x-y|^{2}}{4 u}} \frac{d u}{u},
$$


where $\widetilde{\alpha}=\left(\widetilde{\alpha}_{1}, \alpha^{\prime}\right)$ as before, and the sum is taken over $\widetilde{\alpha}_{1}=0, \ldots, \alpha_{1}$. Using (2.8), we see that this expression equals

$$
\begin{aligned}
& (-1)^{k} \pi^{-\frac{n+1}{2}} 2^{-|\widetilde{\alpha}|-n-1}\left(\begin{array}{c}
\alpha_{1} \\
\widetilde{\alpha}_{1}
\end{array}\right) e^{-x_{1}-y_{1}} t \\
& \quad \times \int_{0}^{+\infty} u^{-\frac{1+|\widetilde{\alpha}|+n}{2}} H_{\widetilde{\alpha}}\left(\frac{x-y}{2 \sqrt{u}}\right) e^{-u} e^{-\frac{t^{2}+|x-y|^{2}}{4 u}} \frac{d u}{u} .
\end{aligned}
$$

We now apply (2.5) to the integral here. It follows that for large $|x-y|$, quantity (5.3) equals

$$
\begin{aligned}
& (-1)^{k}(2 \pi)^{-\frac{n}{2}} 2^{-\frac{|\widetilde{\alpha}|}{2}}\left(\begin{array}{c}
\alpha_{1} \\
\widetilde{\alpha}_{1}
\end{array}\right) t\left(t^{2}+|x-y|^{2}\right)^{-\frac{1+\mid \widetilde{\alpha}+n}{4}-\frac{1}{4}} e^{-x_{1}-y_{1}-\sqrt{t^{2}+|x-y|^{2}}} \\
& \quad \times H_{\widetilde{\alpha}}\left(\frac{x-y}{\sqrt{2}\left(t^{2}+|x-y|^{2}\right)^{1 / 4}}\right)\left(1+O\left(\frac{1}{\sqrt{t^{2}+|x-y|^{2}}}\right)\right) .
\end{aligned}
$$

Again, we consider points $x$ for which $x_{1}>0$ is large and $x^{\prime} / \sqrt{x_{1}}$ stays bounded. Further, we assume $|y|<1$ and restrict $t$ by $\sqrt{x_{1}}<t<2 \sqrt{x_{1}}$. Some Taylor expansions then lead to $|x-y|=x_{1}\left(1+O\left(x_{1}^{-1}\right)\right)$ and

$$
\begin{aligned}
\sqrt{t^{2}+|x-y|^{2}} & =|x|\left(1-\frac{x \cdot y}{|x|^{2}}+\frac{t^{2}}{2|x|^{2}}+O\left(\frac{1}{|x|^{2}}\right)\right) \\
& =|x|-y_{1}+\frac{t^{2}}{2 x_{1}}+O\left(\frac{1}{\sqrt{x_{1}}}\right) \\
& =x_{1}\left(1+O\left(x_{1}^{-1}\right)\right),
\end{aligned}
$$

as $x_{1} \rightarrow \infty$.

Using (5.5), we conclude that expression (5.4) now equals

$$
\begin{aligned}
& (-1)^{k}(2 \pi)^{-\frac{n}{2}} 2^{-\frac{\mid \widetilde{\alpha}}{2}}\left(\begin{array}{l}
\alpha_{1} \\
\widetilde{\alpha}_{1}
\end{array}\right) t x_{1}^{-\frac{\mid \widetilde{\alpha}+n+2}{2}} e^{-x_{1}-|x|-\frac{t^{2}}{2 x_{1}}} \\
& \times H_{\widetilde{\alpha}_{1}}\left(\sqrt{\frac{x_{1}}{2}}\right) H_{\alpha^{\prime}}\left(\frac{x^{\prime}}{\sqrt{2 x_{1}}}\right)\left(1+O\left(\frac{1}{\sqrt{x_{1}}}\right)\right),
\end{aligned}
$$

as $x_{1} \rightarrow \infty$. We can replace the Hermite polynomial $H_{\widetilde{\alpha}_{1}}\left(\sqrt{x_{1} / 2}\right)$ here by its leading term, which is $2^{\widetilde{\alpha}_{1} / 2} x_{1}^{\widetilde{\alpha}_{1} / 2}$. Since $|\widetilde{\alpha}|=\widetilde{\alpha}_{1}+\left|\alpha^{\prime}\right|$, this will make the exponents of 2 and $x_{1}$ in (5.6) independent of $\widetilde{\alpha}_{1}$. When we then sum in $\widetilde{\alpha}_{1}=0, \ldots, \alpha_{1}$, the binomial coefficients in (5.6) will sum up to $2^{\alpha_{1}}$, and as a result,

$$
\begin{aligned}
\partial_{x}^{\alpha} P_{t}(x, y)= & (-1)^{k}(2 \pi)^{-\frac{n}{2}} 2^{\alpha_{1}-\frac{\left|\alpha^{\prime}\right|}{2}} t x_{1}^{-\frac{\left|\alpha^{\prime}\right|+n+2}{2}} e^{-x_{1}-|x|-\frac{t^{2}}{2 x_{1}}} \\
& \times H_{\alpha^{\prime}}\left(\frac{x^{\prime}}{\sqrt{2 x_{1}}}\right)\left(1+O\left(\frac{1}{\sqrt{x_{1}}}\right)\right) .
\end{aligned}
$$

Next, we integrate against $f(y) d \mu(y)$ with $f=\chi_{B(0,1)} / \mu(B(0,1))$. Summing also over $\alpha$, we get

$$
D_{x} P_{t} f(x)=(-1)^{k}(2 \pi)^{-\frac{n}{2}} t x_{1}^{-\frac{n+2}{2}} e^{-x_{1}-|x|-\frac{t^{2}}{2 x_{1}}}
$$




$$
\times \sum_{\alpha} 2^{\alpha_{1}-\frac{\left|\alpha^{\prime}\right|}{2}} a_{\alpha} x_{1}^{-\frac{\left|\alpha^{\prime}\right|}{2}} H_{\alpha^{\prime}}\left(\frac{x^{\prime}}{\sqrt{2 x_{1}}}\right)\left(1+O\left(\frac{1}{\sqrt{x_{1}}}\right)\right) .
$$

For large $x_{1}$, the largest terms in the sum here are those where $\left|\alpha^{\prime}\right|$ takes its minimal value $k-q$, so that $\alpha_{1}=q$. We introduce the nonzero polynomial

$$
P\left(z^{\prime}\right)=(-1)^{k}(2 \pi)^{-\frac{n}{2}} 2^{\frac{3 q-k}{2}} \sum_{\left|\alpha^{\prime}\right|=k-q} a_{\left(q, \alpha^{\prime}\right)} H_{\alpha^{\prime}}\left(z^{\prime}\right) .
$$

Since $x^{\prime} / \sqrt{2 x_{1}}$ stays bounded, it follows that

$$
t^{k} D_{x} P_{t} f(x)=e^{-x_{1}-|x|-\frac{t^{2}}{2 x_{1}}} t^{k+1} x_{1}^{-\frac{k-q+n+2}{2}}\left(P\left(\frac{x^{\prime}}{\sqrt{2 x_{1}}}\right)+O\left(\frac{1}{\sqrt{x_{1}}}\right)\right) .
$$

We can now integrate in $t$ and conclude that

$$
\left(\mathcal{G}_{D} f(x)\right)^{2} \gtrsim e^{-4 x_{1}} \int_{\sqrt{x_{1}}}^{2 \sqrt{x_{1}}} e^{-\frac{t^{2}}{x_{1}}} t^{2 k+2} \frac{d t}{t} x_{1}^{-(k-q+n+2)}\left(P\left(\frac{x^{\prime}}{\sqrt{2 x_{1}}}\right)^{2}+O\left(\frac{1}{\sqrt{x_{1}}}\right)\right) .
$$

The integral here is of order of magnitude $x_{1}^{k+1}$, and so

$$
\mathcal{G}_{D} f(x) \gtrsim e^{-2 x_{1}} x_{1}^{\frac{q-n-1}{2}}\left(\left|P\left(\frac{x^{\prime}}{\sqrt{2 x_{1}}}\right)\right|+O\left(\frac{1}{\sqrt{x_{1}}}\right)\right) .
$$

To obtain (5.2), it is now enough to let $B$ be a closed ball in $\mathbb{R}^{n-1}$ in which the polynomial $P$ does not vanish.

This ends the proof of Theorem 1.2(ii).

\section{Sharp Estimates for $\frac{\partial^{k}}{\partial t^{k}} p_{t}$}

This section is a preparation for the proof of Theorem 1.3.

We take $k$ derivatives with respect to $t$ of

$$
p_{t}(x, y)=(4 \pi)^{-\frac{n}{2}} e^{-x_{1}-y_{1}} t^{-\frac{n}{2}} e^{-t-\frac{|x-y|^{2}}{4 t}} .
$$

Those derivatives that fall on the last exponential here will produce factors $\frac{|x-y|^{2}}{4 t^{2}}-1$. The derivative

$$
\frac{\partial}{\partial t}\left[\frac{|x-y|^{2}}{4 t^{2}}-1\right]=-\frac{|x-y|^{2}}{2 t^{3}}
$$

will also appear. We see that

$$
\frac{\partial^{k}}{\partial t^{k}} p_{t}(x, y)=q_{k} p_{t}(x, y)
$$

where the factor $q_{k}$ is given by

$$
q_{k}=Q_{k}\left(\frac{|x-y|^{2}}{4 t^{2}}-1, \frac{1}{t}, \frac{|x-y|^{2}}{2 t^{3}}\right)
$$

for a polynomial $Q_{k}$ in three variables, whose coefficients depend only on $n$ and $k$. 
To estimate $q_{k}$, we examine the terms of this polynomial. Consider a term obtained by letting exactly $m$ of the $k$ differentiations fall on the exponential $e^{-t-|x-y|^{2} / 4 t}$. If, moreover, the number of differentiations falling on a power of $\frac{|x-y|^{2}}{4 t^{2}}-1$ is $p$, the resulting term of $Q_{k}$ will contain $p$ factors $-\frac{|x-y|^{2}}{2 t^{3}}$ and $m-p$ factors $\frac{|x-y|^{2}}{4 t^{2}}-1$. The remaining $k-m-p$ differentiations will produce factors $-t^{-1}$. We conclude that $q_{k}$ is a sum of expressions of the form

$$
C_{m, p}\left(\frac{|x-y|^{2}}{4 t^{2}}-1\right)^{m-p}\left(-\frac{|x-y|^{2}}{2 t^{3}}\right)^{p}(-t)^{-(k-m-p)}
$$

with $C_{m, p}>0$. Here, $p+m \leq k$ and $p \leq m$; thus, $2 p \leq k$ so that $p \leq\left[\frac{k}{2}\right]$. This implies the upper estimate

$$
\left|\frac{\partial^{k}}{\partial t^{k}} p_{t}(x, y)\right| \lesssim \sum\left|\frac{|x-y|^{2}}{4 t^{2}}-1\right|^{m-p}|x-y|^{2 p} t^{-k+m-2 p} p_{t}(x, y),
$$

the sum taken over $0 \leq p \leq\left[\frac{k}{2}\right]$ and $0 \leq m \leq k-p$.

We also need a lower estimate. Let $\eta>0$ be large and define

$$
\Sigma_{\eta}=\left\{x ; \eta-1<x_{1}<\eta \quad \text { and } \sqrt{\eta}<x_{i}<2 \sqrt{\eta}, \quad i=2, \ldots, n\right\} .
$$

Lemma 6.1 For $x \in \Sigma_{\eta},|y|<1$, and

$$
\frac{\eta}{2}\left(1-2 \frac{c_{1}}{\sqrt{\eta}}\right)<t<\frac{\eta}{2}\left(1-\frac{c_{1}}{\sqrt{\eta}}\right)
$$

with $c_{1}=c_{1}(n, k)$ small enough and $\eta$ large enough, one has

$$
(-1)^{\left[\frac{k}{2}\right]} t^{k} \frac{\partial^{k}}{\partial t^{k}} p_{t}(x, y) \gtrsim e^{-2 \eta} \eta^{\frac{k-n}{2}} .
$$

Proof Assuming $x, y$, and $t$ as in the lemma, we can write

$$
t=\frac{\eta}{2}\left(1-\frac{\zeta}{\sqrt{\eta}}\right)
$$

where $c_{1}<\zeta<2 c_{1}$.

Some simple computations will lead to $|x-y|=\eta\left(1+O\left(\eta^{-1}\right)\right)$ and

$$
\frac{|x-y|^{2}}{4 t^{2}}-1=2 \frac{\zeta}{\sqrt{\eta}}\left(1+O\left(\eta^{-\frac{1}{2}}\right)\right) \text { and } \frac{|x-y|^{2}}{2 t^{3}}=\frac{4}{\eta}\left(1+O\left(\eta^{-\frac{1}{2}}\right)\right) \text {, }
$$

as $\eta \rightarrow \infty$. Expression (6.3) thus equals

$$
\begin{gathered}
(-1)^{m-k} C_{m, p}\left(2 \frac{\zeta}{\sqrt{\eta}}\right)^{m-p}\left(\frac{4}{\eta}\right)^{p}\left(\frac{\eta}{2}\right)^{-k+m+p}\left(1+O\left(\eta^{-\frac{1}{2}}\right)\right. \\
=(-1)^{m-k} C_{m, p} 2^{k} \eta^{\frac{m+p}{2}-k} \zeta^{m-p}\left(1+O\left(\eta^{-\frac{1}{2}}\right)\right) .
\end{gathered}
$$

If we fix a small $c_{1}>0$ and take $\eta>c_{1}^{-1}$, the product $2^{k} \eta^{\frac{m+p}{2}-k} \zeta^{m-p}$ will be maximal when $m+p$ takes its largest possible value $k$ and also $m-p$ is as small as possible. This means that $p=\left[\frac{k}{2}\right]$ and $m=k-\left[\frac{k}{2}\right]$. Moreover, if $c_{1}$ is small enough and $\eta$ large 
enough, these values of $m$ and $p$ will make expression (6.6) much larger than any other admissible values of $m$ and $p$, in modulus. We conclude that then

$$
(-1)^{\left[\frac{k}{2}\right]} q_{k} \sim \eta^{-\frac{k}{2}} \zeta^{k-2\left[\frac{k}{2}\right]} \sim \begin{cases}\eta^{-\frac{k}{2}}, & k \text { even, } \\ c_{1} \eta^{-\frac{k}{2}}, & k \text { odd }\end{cases}
$$

We also need an estimate of the value of $p_{t}(x, y)$ for these $x, y, t$, and write the sum of the exponents of $e$ in (6.1) as

$$
\begin{aligned}
-x_{1} & -y_{1}-t-\frac{|x-y|^{2}}{4 t} \\
& =-\eta+O(1)-\frac{\eta}{2}+\frac{\zeta \sqrt{\eta}}{2}-\frac{\eta^{2}\left(1+O\left(\eta^{-1}\right)\right)}{2 \eta(1-\zeta / \sqrt{\eta})} \\
& =-\eta+O(1)-\frac{\eta}{2}+\frac{\zeta \sqrt{\eta}}{2}-\frac{\eta}{2}\left(1+\frac{\zeta}{\sqrt{\eta}}+O\left(\eta^{-1}\right)\right) \\
& =-2 \eta+O(1) .
\end{aligned}
$$

Thus, (6.1) implies that

$$
p_{t}(x, y) \sim e^{-2 \eta} t^{-\frac{n}{2}} \text {. }
$$

We now combine this with (6.2) and (6.7), and take $\eta$ large after fixing a small $c_{1}$. Then (6.5) follows.

\section{Proof of Theorem 1.3}

For the local parts of the operators in Theorem 1.3, one can use the method of localization, since $\mathbb{R}^{(n, v)}$ has the local doubling property. Standard vector-valued singular integral theory then gives the weak type $(1,1)$ of the local parts of $g_{k}, h_{k}$ and $H_{k}$.

The parts at infinity of these operators can all be estimated by the method used for Theorem 1.1, in the following way.

Consider first the part at infinity of $h_{k}$, given by

$$
h_{k}^{\infty}(f)(x)=\left(\int_{0}^{+\infty}\left|t^{k} \int_{|x-y|>1} \frac{\partial^{k}}{\partial t^{k}} p_{t}(x, y) f(y) d \mu(y)\right|^{2} \frac{d t}{t}\right)^{\frac{1}{2}} .
$$

Using Minkowski's integral inequality, we get

$$
h_{k}^{\infty}(f)(x) \leq \int_{|x-y|>1}|f(y)|\left(\int_{0}^{+\infty}\left|t^{k} \frac{\partial^{k}}{\partial t^{k}} p_{t}(x, y)\right|^{2} \frac{d t}{t}\right)^{\frac{1}{2}} d \mu(y) .
$$

We estimate the inner integral here.

Lemma 7.1 We have

$$
\left(\int_{0}^{+\infty}\left|t^{k} \frac{\partial^{k}}{\partial t^{k}} p_{t}(x, y)\right|^{2} \frac{d t}{t}\right)^{\frac{1}{2}} \lesssim|x-y|^{\frac{k-n}{2}-\frac{1}{4}} e^{-x_{1}-y_{1}-|x-y|}, \quad|x-y|>1 .
$$


Since this lemma implies a kernel estimate like (2.3) with $q$ replaced by $k+1 / 2$, it will allow us to argue as in Section 3 using the operator $\widetilde{\mathcal{V}}_{k+1 / 2}$. In this way, the weak type $(1,1)$ of $h_{1}$ and also (1.5) follow from Lemma 7.1.

Proof of Lemma 7.1 Combining (6.4) and (1.9), we conclude

$(7.1)$

$$
\begin{aligned}
& \int_{0}^{+\infty}\left|t^{k} \frac{\partial^{k}}{\partial t^{k}} p_{t}(x, y)\right|^{2} \frac{d t}{t} \lesssim e^{-2 x_{1}-2 y_{1}-2|x-y|} \\
& \quad \times \sum|x-y|^{4 p} \int_{0}^{+\infty}\left|\frac{|x-y|}{2 t}+1\right|^{2(m-p)}\left|\frac{|x-y|}{2 t}-1\right|^{2(m-p)} t^{2 m-4 p-n} e^{-2 t\left(\frac{|x-y|}{2 t}-1\right)^{2}} \frac{d t}{t},
\end{aligned}
$$

where the sum is taken over $m$ and $p$ such that $0 \leq p \leq\left[\frac{k}{2}\right]$ and $0 \leq m \leq k-p$. In the last integral, we estimate

$$
\left|\frac{|x-y|}{2 t}-1\right|^{2(m-p)} e^{-2 t\left(\frac{|x-y|}{2 t}-1\right)^{2}} \lesssim t^{p-m} e^{-t\left(\frac{|x-y|}{2 t}-1\right)^{2}} .
$$

Changing variables by $t=|x-y| /(2 s)$, we see that the integral in the right-hand side of (7.1) is controlled by

$$
\int_{0}^{+\infty}(s+1)^{2(m-p)}(|x-y| / s)^{m-3 p-n} e^{-\frac{1}{2}|x-y|(s-1)^{2} / s} \frac{d s}{s} .
$$

If restricted to the interval $1 / 2<s<2$, this integral will be of order of magnitude $\mid x-$ $\left.y\right|^{m-3 p-n-1 / 2}$. The other parts of the integral are smaller because of the exponential decay. From this, Lemma 7.1 follows.

Let us now consider the part at infinity of $g_{k}, k \geq 1$. It is enough to verify the following estimate:

$$
\left(\int_{0}^{+\infty}\left|t^{k} \frac{\partial^{k}}{\partial t^{k}} P_{t}(x, y)\right|^{2} \frac{d t}{t}\right)^{\frac{1}{2}} \lesssim|x-y|^{-\frac{n+1}{2}} e^{-x_{1}-y_{1}-|x-y|}, \quad|x-y|>1,
$$

since we can then argue as in Section 3. For this, we write the subordination formula as

$$
P_{t}(x, y)=\int_{0}^{+\infty} \phi\left(\frac{t}{2 \sqrt{u}}\right) p_{u}(x, y) \frac{d u}{u} \quad \text { with } \quad \phi(s)=\frac{s}{\sqrt{\pi}} e^{-s^{2}}
$$

and use Minkowski’s integral inequality, getting

$$
\begin{aligned}
\left(\int_{0}^{+\infty}\right. & \left.\left|t^{k} \frac{\partial^{k}}{\partial t^{k}} P_{t}(x, y)\right|^{2} \frac{d t}{t}\right)^{\frac{1}{2}} \\
& \leq \int_{0}^{+\infty}\left\{\left(\int_{0}^{+\infty}\left|\left(\frac{t}{2 \sqrt{u}}\right)^{k} \phi^{(k)}\left(\frac{t}{2 \sqrt{u}}\right)\right|^{2} \frac{d t}{t}\right)^{\frac{1}{2}}\right\} p_{u}(x, y) \frac{d u}{u} \\
& \lesssim \int_{0}^{+\infty} p_{u}(x, y) \frac{d u}{u} .
\end{aligned}
$$


In view of (1.8), the last expression is majorized by constant times

$$
e^{-x_{1}-y_{1}} \int_{0}^{+\infty} u^{-\frac{n}{2}} e^{-u-\frac{|x-y|^{2}}{4 u}} \frac{d u}{u}
$$

By Lemma 2.3(ii), we see that for $|x-y|>1$, this quantity is no larger than constant times $e^{-x_{1}-y_{1}-|x-y|}|x-y|^{-(n+1) / 2}$, and (7.3) follows.

The next lemma will imply the weak type $(1,1)$ of $H_{1}$ and also (1.6).

Lemma 7.2 For $|x-y|>1, t>0$, and $k \geq 1$,

$$
\left|t^{k} \frac{\partial^{k}}{\partial t^{k}} p_{t}(x, y)\right| \lesssim e^{-x_{1}-y_{1}-|x-y|}|x-y|^{\frac{k-n}{2}}
$$

Proof From (6.4) and (1.9), we obtain

$$
\begin{aligned}
\left|t^{k} \frac{\partial^{k}}{\partial t^{k}} p_{t}(x, y)\right| \lesssim e^{-x_{1}-y_{1}-|x-y|} \sum \mid \frac{|x-y|}{2 t}- & \left.1\right|^{m-p}\left(\frac{|x-y|}{2 t}+1\right)^{m-p} \\
& \times|x-y|^{2 p} t^{m-2 p-\frac{n}{2}} e^{-t\left(\frac{|x-y|}{2 t}-1\right)^{2}},
\end{aligned}
$$

the sum taken over $0 \leq p \leq\left[\frac{k}{2}\right], 0 \leq m \leq k-p$. We now use (7.2), where we first take square roots, and write again $t=s|x-y| / 2$. The result will be

$$
\left|t^{k} \frac{\partial^{k}}{\partial t^{k}} p_{t}(x, y)\right| \lesssim e^{-x_{1}-y_{1}-|x-y|} \sum\left(\frac{1}{s}+1\right)^{m-p}|x-y|^{\frac{p+m-n}{2}} s^{\frac{m-3 p-n}{2}} e^{-\frac{1}{4}|x-y| s\left(\frac{1}{s}-1\right)^{2}} .
$$

Here we simply delete the factor $|x-y|$ in the last exponent. Since $p+m \leq k$, we will get at most the quantity in the right-hand side of the lemma, multiplied by a bounded function of $s$.

Lemma 7.2 is proved.

To verify the sharpness parts of Theorem 1.3, we use Lemma 6.1. For $h_{k}$ we get with $x \in \Sigma_{\eta}$

$$
\left(h_{k}\left(\chi_{B(0,1)}\right)(x)\right)^{2} \gtrsim \int_{\eta\left(1-2 c_{1} / \sqrt{\eta}\right) / 2}^{\eta\left(1-c_{1} / \sqrt{\eta}\right) / 2} e^{-4 \eta} \eta^{k-n} \frac{d t}{t} \gtrsim e^{-4 \eta} \eta^{k-n-\frac{1}{2}}=\lambda^{2},
$$

where the equality defines the positive number $\lambda$. Since for $k \geq 2$,

$$
\lambda \mu\left(\Sigma_{\eta}\right) \gtrsim e^{-2 \eta} \eta^{\frac{k-n-\frac{1}{2}}{2}} e^{2 \eta} \eta^{\frac{n-1}{2}}=\eta^{\frac{k}{2}-\frac{3}{4}} \longrightarrow+\infty \text { as } \eta \longrightarrow+\infty,
$$

we conclude that $h_{k}$ is not of weak type $(1,1)$ and that $(1.5)$ is sharp.

The case of $H_{k}$ is even simpler, and this ends the proof of Theorem 1.3.

\section{References}

[1] G. Alexopoulos, An application of homogenization theory to harmonic analysis: Harnack inequalities and Riesz transforms on Lie groups of polynomial growth. Canad. J. Math. 44(1992), 691-727. http://dx.doi.org/10.4153/CJM-1992-042-x

[2] A. Andersson and P. Sjögren, Ornstein-Uhlenbeck theory in finite dimension. Lecture Notes Dept. Math. Sciences, University of Gothenburg, Chalmers, 2012.

[3] J.-P. Anker, Sharp estimates for some functions of the Laplacian on noncompact symmetric spaces. Duke Math. J. 65(1992), 257-297. http://dx.doi.org/10.1215/S0012-7094-92-06511-2 
[4] J.-P. Anker, E. Damek, and C. Yacoub, Spherical analysis on harmonic AN groups. Ann. Scuola Norm. Sup. Pisa Cl. Sci. (4) 23(1996), 643-679.

[5] P. Auscher, T. Coulhon, X.-T. Duong, and S. Hofmann, Riesz transform on manifolds and heat kernel regularity. Ann. Sci. École Norm. Sup. (4) 37(2004), 911-957. http://dx.doi.org/10.1016/j.ansens.2004.10.003

[6] D. Bakry, Étude des transformations de Riesz dans les variétés riemanniennes à courbure de Ricci minorée. In: Séminaire de Probabilités XXI, Lecture Notes in Math., 1247, Springer, Berlin, 1987, pp. 137-172. http://dx.doi.org/10.1007/BFb0077631

[7] G. Carron, T. Coulhon, and A. Hassell, Riesz transform and $L^{p_{-}}$-cohomology for manifolds with Euclidean ends. Duke Math. J. 133(2006), 59-93. http://dx.doi.org/10.1215/S0012-7094-06-13313-6

[8] L. Chen, T. Coulhon, J. Feneuil, and E. Russ, Riesz transform for $1 \leq p \leq 2$ without Gaussian heat kernel bound. J. Geom. Anal. 27(2017), 1489-1514. http://dx.doi.org/10.1007/s12220-016-9728-5

[9] T. Coulhon and X. T. Duong, Riesz transforms for $1 \leq p \leq 2$. Trans. Amer. Math. Soc. 351(1999) 1151-1169. http://dx.doi.org/10.1090/S0002-9947-99-02090-5

[10] T. Coulhon, X.-T. Duong, and X.-D. Li, Littlewood-Paley-Stein functions on complete Riemannian manifolds for $1 \leq p \leq 2$. Studia Math. 154(2003), 37-57. http://dx.doi.org/10.4064/sm154-1-4

[11] T. Coulhon and H.-Q. Li, Estimations inférieures du noyau de la chaleur sur les variétés coniques et transformée de Riesz. Arch. Math. 83(2004), 229-242. http://dx.doi.org/10.1007/s00013-004-1029-8

[12] A. F. M. Elst, D. W. Robinson, and A. Sikora, Riesz transforms and Lie groups of polynomial growth. J. Funct. Anal. 162(1999), 14-51. http://dx.doi.org/10.1006/jfan.1998.3361

[13] E. B. Fabes, C. E. Gutiérrez, and R. Scotto, Weak-type estimates for the Riesz transforms associated with the Gaussian measure. Rev. Mat. Iberoamericana 10(1994), 229-281. http://dx.doi.org/10.4171/RMI/152

[14] G. I. Gaudry, T. Qian, and P. Sjögren, Singular integrals associated to the Laplacian on the affine group $a x+b$. Ark. Math. 30(1992), 259-281. http://dx.doi.org/10.1007/BF02384874

[15] I. S. Gradshteyn and I. M. Ryzhik, Table of integrals, series, and products. 7 th ed. Translation edited and with a preface by Alan Jeffrey and Daniel Zwillinger. Academic Press, Inc., San Diego, CA, 2007.

[16] A. Grigor'yan, Heat kernel and analysis on manifolds. AMS/IP Studies in Advanced Mathematics, 47, American Mathematical Society, Providence, RI, International Press, Boston, MA, 2009. http://dx.doi.org/10.1090/amsip/047

[17] W. Hebisch and T. Steger, Multipliers and singular integrals on exponential growth groups. Math. Z. 245(2003), 37-61. http://dx.doi.org/10.1007/s00209-003-0510-6

[18] H.-Q Li, La transformation de Riesz sur les variétés coniques. J. Funct. Anal. 168(1999), 145-238. http://dx.doi.org/10.1006/jfan.1999.3464

[19] H.-Q. Li and P. Sjögren, Weak type $(1,1)$ for some operators related to the Laplacian with drift on real hyperbolic spaces. Potential Anal. 46(2017), 463-484. http://dx.doi.org/10.1007/s11118-016-9590-x

[20] H.-Q. Li, P. Sjögren, and Y.-R. Wu, Weak type $(1,1)$ of some operators for the Laplacian with drift. Math. Z. 282(2016), 623-633. http://dx.doi.org/10.1007/s00209-015-1555-Z

[21] H.-Q. Li and J.-X. Zhu, A note on "Riesz transform for $1 \leq p \leq 2$ without Gaussian heat kernel bound”. J. Geom. Anal. 28(2018), 1597-1609. http://dx.doi.org/10.1007/s12220-017-9879-z

[22] N. Lohoué, Comparaison des champs de vecteurs et des puissances du laplacien sur une variété riemannienne à courbure non positive. J. Funct. Anal. 61(1985), 164-201. http://dx.doi.org/10.1016/0022-1236(85)90033-3

[23] N. Lohoué, Estimation des fonctions de Littlewood-Paley-Stein sur les variétés riemanniennes à courbure non positive. Ann. Sci. École Norm. Sup. 20(1987), 505-544.

[24] N. Lohoué, Transformées de Riesz et fonctions sommables. Amer. J. Math. 114(1992), 875-922. http://dx.doi.org/10.2307/2374800

[25] N. Lohoué and S. Mustapha, Sur les transformées de Riesz sur les espaces homogènes des groupes de Lie semi-simples. Bull. Soc. Math. France 128(2000), 485-495.

[26] N. Lohoué and S. Mustapha, Sur les transformées de Riesz dans le cas du Laplacien avec drift. Trans. Amer. Math. Soc. 356(2004), 2139-2147. http://dx.doi.org/10.1090/S0002-9947-04-03159-9

[27] S. Pérez and F. Soria, Operators associated with the Ornstein-Uhlenbeck semigroup. J. Lond. Math. Soc. (2) 61(2000), 857-871. http://dx.doi.org/10.1112/S0024610700008917

[28] P. Sjögren and M. Vallarino, Boundedness from $H^{1}$ to $L^{1}$ of Riesz transforms on a Lie group of exponential growth. Ann. Inst. Fourier 58(2008), 1117-1151. 
[29] E. M. Stein, Topics in harmonic analysis related to the Littlewood-Paley theory. Annals of Mathematics Studies, 63, Princeton University Press, Princeton, NJ, 1970.

[30] E. M. Stein and N. J. Weiss, On the convergence of Poisson integrals. Trans. Amer. Math. Soc. 140(1969), 35-54. http://dx.doi.org/10.2307/1995121

School of Mathematical Sciences, Fudan University, 220 Handan Road, Shanghai 200433, People’s Republic of China

e-mail: hongquan_li@fudan.edu.cn hong_quanli@yahoo.fr

Mathematical Sciences, University of Gothenburg, and Mathematical Sciences, Chalmers, SE-412 96 Göteborg, Sweden

e-mail: peters@chalmers.se 